\title{
Lateral Heterogeneity and Azimuthal Anisotropy of the Upper Mantle: Love and Rayleigh Waves 100-250 s
}

\author{
Toshiro Tanimoto and Don L. Anderson
}

Seismological Laboratory, California Institute of Technology, Pasadena

\begin{abstract}
The lateral heterogeneity and apparent anisotropy of the upper mantle are studied by measuring Rayleigh and Love wave phase velocities in the period range 100-250 s. Spherical harmonic descriptions of the lateral heterogeneity are obtained for order and degree up to $1=m=10$. Slow regions are evident at the East Pacific Rise, northeast Africa, Tibet, Tasman Sea, southwestern North America, and triple junctions in the northern Atlantic and Indian oceans. Fast regions occur in Australia, western Pacific, and the southern Atlantic. These features are also found by a completely different analysis based on the Backus-Gilbert method. The Backus-Gilbert method also shows that the obtained phase velocities are averaged values within an area of about $2000-\mathrm{km}$ radius and the errors are about $1 \%$ of the phase velocity in the zeroth-order spherically symmetric earth. Inversion for azimuthal dependence shows that for low angular order the fast phase velocity directions seem to correlate well with the plate motion vectors. However, resolution analysis by the Backus-Gilbert method shows that the current data do not have enough resolution for everywhere on the globe. Only a few regions currently have adequate azimuthal coverage. Thus confirmation of the above correlation requires a more complete data set.
\end{abstract}

\section{INTRODUCTION}

There are two approaches which have been used for the inversion of long-period ( $>100 \mathrm{~s}$ ) seismograms for lateral heterogeneity of the earth. One uses waveform inversion and the other uses phase velocity and/or group velocity measurements. The former [Woodhouse, 1984; Woodhouse and Dziewonski, $1984]$ is probably the most direct approach and fully utilizes the information contained in the seismograms, although the current formulation by Woodhouse and Dziewonski [1984] is such that it only takes account of phase perturbation along a path. A disadvantage of this approach is the large storage and computation time required. The velocity method [e.g., $\mathrm{Naka}$ nishi and Anderson, 1982, 1983, 1984] uses only the phase information of the data; the amplitude information is discarded. The measured velocity is the data that the model should satisfy. Measurement of multiplet locations [Silver and Jordan, 1981; Masters et al., 1982] is a variant of this approach, since essentially the same information is used. The velocity method requires relatively little storage and computation time.

There are other advantages and disadvantages associated with the two methods. Waveform inversion can treat higher modes easily. It is difficult to isolate modes for phase velocity and group velocity measurements. For the retrieval of azimuthal anisotropy the velocity measurement approach has the advantage, at least at present, since it is very easy to include in the inversion scheme. Partial derivatives with respect to anisotropy, other than transverse isotropy, have not been derived. These are required in the waveform inversion approach.

The aims of this paper are to obtain (1) the lateral variation of phase velocity of long-period surface waves (100-250 s) and (2) the azimuthal dependence of phase velocity. We use two methods to do this: the spherical harmonic approach and the Backus-Gilbert approach. First, we show the results by the former method and discuss what the robust features are in the

Copyright 1985 by the American Geophysical Union.

Paper number 4B1309.

0148-0227/85/004B-1309\$05.00 solutions in the singular value decomposition approach. In the following section, we attempt to answer the problems of resolution and error by the Backus-Gilbert approach.

\section{The SPHERICAL HARMONIC APPROACH}

Let $t$ be the travel time of the phase at frequency $\omega$ from the source to the receiver and $v(\theta, \varphi, \Psi)$ be the phase velocity as a function of position $(\theta, \varphi)$ and azimuth $\Psi$. We measure $\Psi$ clockwise from north. We have

$$
t=\int_{S}^{R} \frac{d s}{v(\theta, \varphi, \Psi)}
$$

where the right-hand side is the line integral from the source $S$ to the receiver $R$. To first order, this is a stationary quantity, and the wave path (this line integral) is assumed to be along the great circular path containing $S$ and $R$.

Phase velocity at $(\theta, \varphi), v(\theta, \varphi, \Psi)$ can be written as

$$
v(\theta, \varphi, \Psi)=v_{0}+v_{h}(\theta, \varphi)+v_{a}(\theta, \varphi, \Psi)
$$

where $v_{0}$ is the spherically averaged phase velocity and $v_{h}$ and $v_{a}$ are the deviations due to the heterogeneity and anisotropy. We assume $\left|v_{h}\right|,\left|v_{a}\right| \ll\left|v_{0}\right|$. Following Backus [1965] and Smith and Dahlen [1973], $v_{a}$ can be expressed as

$$
v_{a}(\theta, \varphi, \Psi)=a \cos 2 \Psi+b \sin 2 \Psi+c \cos 4 \Psi+d \sin 4 \Psi
$$

We substitute (2) and (3) in (1), expand the denominator, and obtain

$$
\begin{aligned}
t= & \frac{\Delta_{S R}}{v_{0}}-\frac{1}{v_{0}^{2}} \int_{S}^{R} v_{h} d s \\
& +\frac{1}{v_{0}^{2}} \int_{S}^{R}(a \cos 2 \Psi+b \sin 2 \Psi+c \cos 4 \Psi+d \sin 4 \Psi) d s
\end{aligned}
$$

where $\Delta_{S R}$ is the distance between $S$ and $R$. Denoting $\Delta_{S R}$ $-v_{0} t=\delta \varphi$ and expanding $v_{h}, a, b, c$, and $d$ in terms of spheri- 
TABLE 1. List of Earthquakes (in 1980) Used

\begin{tabular}{clclcll}
\hline No. & Date & Time & Latitude & Longitude & $M_{s}$ & \multicolumn{1}{c}{ Region } \\
\hline 1 & Jan. 1 & $1642: 40.0$ & $38.815^{\circ} \mathrm{N}$ & $27.780^{\circ} \mathrm{W}$ & 6.7 & Azores \\
3 & Feb. 7 & $1049: 16.0$ & $54.158^{\circ} \mathrm{S}$ & $158.890^{\circ} \mathrm{E}$ & 6.5 & Macquarie Islands \\
4 & Feb. 23 & $0551: 03.2$ & $43.530^{\circ} \mathrm{N}$ & $146.753^{\circ} \mathrm{E}$ & 7.0 & Kurile Islands \\
7 & March 24 & $0359: 51.3$ & $52.969^{\circ} \mathrm{N}$ & $167.670^{\circ} \mathrm{W}$ & 6.9 & Fox Islands \\
8 & June 9 & $0328: 18.9$ & $32.220^{\circ} \mathrm{N}$ & $114.985^{\circ} \mathrm{W}$ & 6.4 & Cal-Mex Border \\
12 & July 8 & $2319: 19.8$ & $12.410^{\circ} \mathrm{S}$ & $166.381^{\circ} \mathrm{E}$ & 7.5 & Santa Cruz Island \\
14 & July 14 & $1615: 01.7$ & $29.273^{\circ} \mathrm{S}$ & $177.154^{\circ} \mathrm{W}$ & 6.6 & Kermadec \\
16 & July 29 & $0311: 56.3$ & $13.101^{\circ} \mathrm{S}$ & $166.338^{\circ} \mathrm{W}$ & 6.7 & Vanuatu Islands \\
19 & Oct. 10 & $1225: 23.5$ & $36.195^{\circ} \mathrm{N}$ & $1.354^{\circ} \mathrm{E}$ & 7.3 & Algeria \\
21 & Oct. 25 & $0700: 07.9$ & $21.982^{\circ} \mathrm{S}$ & $170.025^{\circ} \mathrm{E}$ & 6.7 & Loyalty Island \\
22 & Oct. 25 & $1100: 05.1$ & $21.890^{\circ} \mathrm{S}$ & $169.853^{\circ} \mathrm{E}$ & 7.2 & Loyalty Island \\
24 & Nov. 8 & $1027: 34.0$ & $41.117^{\circ} \mathrm{N}$ & $124.253^{\circ} \mathrm{W}$ & 7.2 & N. California \\
26 & Nov. 23 & $1834: 53.8$ & $40.914^{\circ} \mathrm{N}$ & $15.366^{\circ} \mathrm{E}$ & 6.9 & Italy \\
27 & Dec. 17 & $1621: 58.8$ & $49.479^{\circ} \mathrm{N}$ & $129.496^{\circ} \mathrm{W}$ & 6.8 & Vancouver Island \\
28 & Dec. 31 & $1032: 11.0$ & $46.060^{\circ} \mathrm{N}$ & $151.453^{\circ} \mathrm{E}$ & 6.5 & Kurile Islands \\
\hline
\end{tabular}

cal harmonics, we get

$$
\begin{aligned}
v_{0} \delta \varphi= & \sum_{l m} v_{h l m} \int_{S}^{R} Y_{l}^{m}(\theta, \varphi) d s+\sum_{l m} a_{l m} \int_{S}^{R} Y_{l}^{m}(\theta, \varphi) \cos 2 \Psi d s \\
& +\sum_{l m} b_{l m} \int_{S}^{R} Y_{l}^{m}(\theta, \varphi) \sin 2 \Psi d s \\
& +\sum_{l m} c_{l m} \int_{S}^{R} Y_{l}^{m}(\theta, \varphi) \cos 4 \Psi d s \\
& +\sum_{l m} d_{l m} \int_{S}^{R} Y_{l}^{m}(\theta, \varphi) \sin 4 \Psi d s
\end{aligned}
$$

where $v_{h l m}$, and $a_{l m}, b_{l m}, c_{l m}$, and $d_{l m}$ are spherical harmonic coefficients of $v_{h}, a, b, c$, and $d$. The left-hand side, $v_{0} \delta \varphi$, is determined from the observation and we solve systems of equations like (4) for $v_{h l m}, a_{l m}, b_{l m}, c_{l m}$, and $d_{l m}$. Note that incorporation of anisotropy $2 \Psi$ and $4 \Psi$ increases the number of unknowns by a factor of 5 .

For convenience in later sections we write the system of (4) as

$$
A x=B
$$

where

$$
\begin{gathered}
x=\left(v_{h 00}, v_{h 10}, \cdots, a_{00}, \cdots, b_{00}, \cdots, c_{00}, \cdots, d_{00}, \cdots\right)^{T} \\
B=\left(v_{0} \delta \varphi, \cdots\right)^{T}
\end{gathered}
$$

and the elements of $A$ are given by line integrals in (4).

\section{Data}

Phase velocities measured for 15 earthquakes in 1980 (Table 1) from the records of the International Deployment of Accelerometers (IDA) and the Global Digital Seismograph Network (GDSN) are used in this study. The single-station method which is used for the measurement is explained in detail by Nakanishi and Anderson [1984]. Source parameters used for the measurement are tabulated in Table 2 [Nakanishi and Kanamori, 1984]. The data set is slightly larger than that of Nakanishi and Anderson [1983, 1984]. Only R2 and R3 or G2 and G3 are used for the analysis. Rayleigh and Love waves at periods of $100,150,200$, and $250 \mathrm{~s}$ are analyzed, and the number of data used in each case is given in Table 3 . The number of data for Love waves is less than that of Rayleigh waves since IDA data are restricted to the vertical component.

Figures 1 and 2 show the path coverage of Rayleigh and Love waves at $200 \mathrm{~s}$. Overall patterns are very similar at different periods. In both Figures 1 and 2 the top figure shows the number of surface wave rays that go through each $20^{\circ} \times 20^{\circ}$ block. The bottom figure shows the azimuthal coverage of rays in each block. The azimuth of a ray can change in a block; thus a mean azimuth is calculated for each ray in a block and shown here.

It is clear from Figures 1 and 2 that coverage of the polar regions is not good but the area near polar regions is smaller for a given $\left(20^{\circ} \times 20^{\circ}\right)$ block size. Path coverage per unit area is better than shown in Figures 1 and 2. Good path coverages near the Mediterranean, Japan, South Pacific, and northern Atlantic Ocean correspond to the locations or the antipodes of earthquakes or stations. Spherical harmonic coefficients are mainly controlled by the regions with good coverage. The results in the poorly covered region are essentially extrapolations and should be treated with care.

Overall, path coverage is good, although the azimuthal coverage in some blocks is not. This causes a problem, i.e., trade-off, when azimuthal dependence of phase velocity is incorporated in the inversion. This will be discussed later.

\section{ANALYSIS}

\section{Solution Behavior}

First, we solved the system of (5) by the conventional least squares approach, i.e.,

$$
x=\left(A^{T} A\right)^{-1} A^{T} B
$$

TABLE 2. Source Parameters of the Earthquakes Used

\begin{tabular}{rccccrr}
\hline No. & $\begin{array}{c}\text { Depth, } \\
\mathrm{km}\end{array}$ & $\begin{array}{c}\tau, \\
\mathrm{s}\end{array}$ & $\begin{array}{c}M_{\mathrm{o}}\left(\times 10^{26}\right), \\
\mathrm{dyn} \mathrm{cm}\end{array}$ & $\begin{array}{c}\delta, \\
\mathrm{deg}\end{array}$ & $\begin{array}{c}\lambda, \\
\mathrm{deg}\end{array}$ & \multicolumn{1}{c}{$\begin{array}{r}\varphi, \\
\mathrm{deg}\end{array}$} \\
\hline 1 & 9.75 & 17.2 & 2.38 & 86.2 & 3.0 & -31.0 \\
3 & 9.75 & 29.7 & 1.9 & 84.0 & 0.0 & -70.0 \\
4 & 43.0 & 19.3 & 6.31 & 70.0 & 89.2 & 27.0 \\
7 & 33.0 & 30.1 & 2.95 & 60.0 & 88.1 & 53.3 \\
8 & 9.75 & 15.4 & 0.465 & 90.0 & 180.0 & 140.1 \\
12 & 33.0 & 51.4 & 21.5 & 59.0 & 93.1 & 170.0 \\
14 & 43.0 & 18.0 & 1.38 & 70.0 & 82.9 & 10.0 \\
16 & 43.0 & 19.1 & 1.57 & 54.0 & 93.5 & 160.0 \\
19 & 9.75 & 30.2 & 4.89 & 54.0 & 81.8 & 225.0 \\
21 & 33.0 & 38.7 & 9.30 & 74.0 & 93.2 & 143.0 \\
22 & 33.0 & 46.8 & 29.2 & 73.0 & 88.2 & 142.0 \\
24 & 16.0 & 31.7 & 10.3 & 90.0 & 0.0 & 49.8 \\
26 & 9.75 & 44.7 & 2.84 & 63.0 & 275.8 & -43.0 \\
27 & 9.75 & 26.2 & 1.54 & 90.0 & 180.0 & -37.1 \\
28 & 33.0 & 27.8 & 2.90 & 68.0 & 89.6 & 28.3 \\
\hline
\end{tabular}

Here $\tau$ is the rise time, $M_{0}$ seismic moment, $\delta$ dip angle, $\lambda$ slip angle, and $\varphi$ strike, measured clockwise from north. 
TABLE 3. Number of Data Used for Analysis

\begin{tabular}{ccc}
\hline $\begin{array}{c}\text { Period, } \\
\mathrm{s}\end{array}$ & Rayleigh & Love \\
\hline 100 & 497 & 369 \\
150 & 562 & 385 \\
200 & 577 & 380 \\
250 & 557 & 356 \\
\hline
\end{tabular}

The solution $x$ becomes very large in some cases. Phase velocity variations exceeded $10 \%$, which suggests that considerable errors are mapped to a solution due to the near singularity of $\left(A^{T} A\right)$. At this point we adopted the singular decomposition approach (SVD).

In $\mathrm{SVD}, \boldsymbol{A}$ is decomposed as

$$
A=U \Lambda V^{T}
$$

where $U$ and $V$ are orthogonal matrices and $\Lambda$ is a diagonal matrix whose elements are eigenvalues of $A$. Then $\left(A^{T} A\right)^{-1}$ can be written

$$
\left(A^{T} A\right)^{-1}=V \Lambda^{-2} V^{T}
$$

We eliminate some of the smaller eigenvalues in $\Lambda$ in order to stabilize the solution $x$. The question then arises: how does the solution change as the number of eigenvalues in $\Lambda$ kept in $\left(A^{T} A\right)^{-1}$ (hereafter $p$ ) is changed.

Figure 3 shows the solution behavior of the inversion of
Rayleigh waves at $200 \mathrm{~s}$ for heterogeneity with $l \mathrm{max}=10$. The upper part of Figure 3 shows the solution norm (crosses) and the residual norm (stars). The horizontal axis gives the number of eigenvalues kept $(p)$, and the increment is taken to be 2 . The solution norm is defined as the Eulidean norm of the vector $x$, i.e.,

$$
\|x\|=\left(\sum_{i} x_{i}^{2}\right)^{1 / 2}
$$

where $x^{T}=\left(x_{1}, x_{2}, \cdots\right)$. The residual norm is defined as the Euclidean norm of $A x-B$.

As $p$ is increased, the residual norm decreases and the resolution becomes better while the solution norm increases. The lower part of Figure 3 shows the behavior of some of the solutions. Each solution is normalized for plotting purposes. Some of the features shown in this figure are as follows:

1. There are no drastic changes in the residual and solution norms as $p$ is increased.

2. Whenever there is a small jump in residual norm and solution norm, the solutions change drastically (for example, see around $p=19-23,63-65,71-73$, and 109-111).

3. Solution behaviour is especially erratic from $p=113$ to $p=121$ (maximum), which suggests that considerable errors are being mapped into the solution by incorporating these smallest few eigenvalues.

However, even the solution for $p=101$ showed unrealisti-

\begin{tabular}{|c|c|c|c|c|c|c|c|c|c|c|c|c|c|c|c|c|c|}
\hline \multirow{2}{*}{\multicolumn{2}{|c|}{$\begin{array}{l}155^{\circ} 9 \\
43^{4}\end{array}$}} & \multicolumn{2}{|c|}{$22+2 z$} & 26 & $8 z$ & 120 & 14 & 13 & 13 & 15 & 16 & & 16 & 18 & 14 & \multicolumn{2}{|c|}{118} \\
\hline & & 32 & 38 & 33 & 32 & 41 & 410 & 38 & 66 & 159 & 44 & 38 & 38. & & 38 & 46 & $4 z$ \\
\hline & & 73 & 110 & 69 & 71 & 89 & 170 & 72 & 57 & 86 & 132 & 139 & 63 & 100 & 71 & 92 & 94 \\
\hline 107 & 56 & 44 & 49 & 67 & 597 & 198 & 93 & 41 & 42 & 61 & 58 & 92 & & 45 & 67 ? & 103 & $315 \theta$ \\
\hline 64 & 58 & 46 & 37 & 52 & 86 & 7 & 93. & 85 & 64 & 55 & 43 & 37 & 59 & 94 & 73 & 194 & 99 \\
\hline 51 & 67 & 60 & 76 & 63 & 57 & 66 & 100 & 246 & 102 & 66 & 47 & 48 & 58 & 142 & 108 & 77 & 49 \\
\hline 43 & 73 & 196 & 101 & 72 & 74 & 71 & 98 & 1,15 & 145 & 95 & 73 & 歹2 & 73 & 63 & 79 & 93 & 56 \\
\hline 56 & 68 & 63 & 36 & 42 & 39 & 39 & 51 & 68 & 55 & 47 & 46 & 44 & 36 & 36 & -40 & 55 & 50 \\
\hline 17 & 20 & 24 & 25 & 24 & 23 & 22 & 15 & $e_{14}^{2}$ & 19 & 20 & 25 & 27 & 30 & 30 & 27 & 20 & 14 \\
\hline
\end{tabular}
cally large peaks and troughs, $45 \%$, in the phase velocity variation map. Phase velocity variation of this order if present,

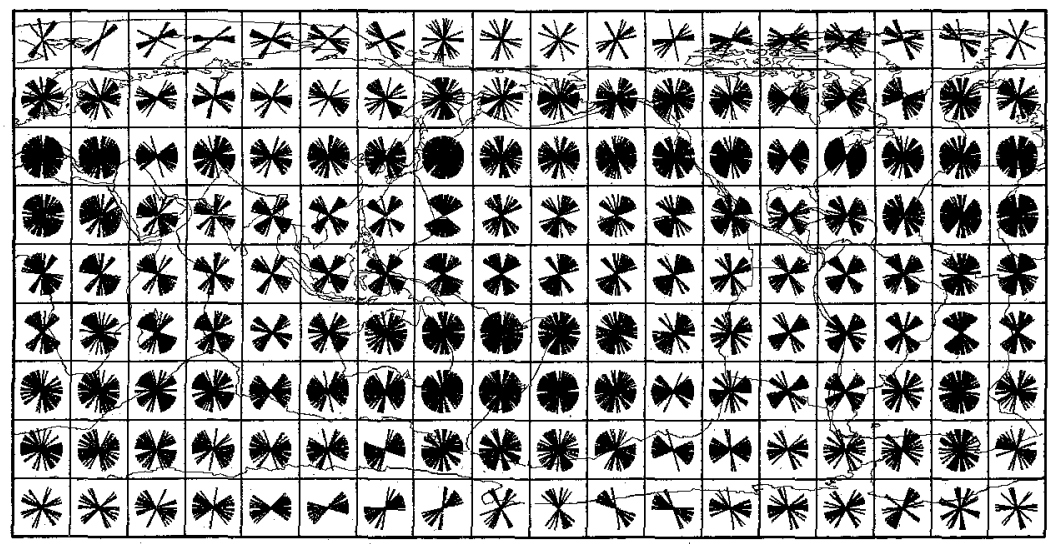

Fig. 1. (Top) The number of surface wave rays in each $20^{\circ} \times 20^{\circ}$ block. (Bottom) The azimuthal coverage of rays in each block. This is for Rayleigh wave at $200 \mathrm{~s}$. Path coverages are similar for 100, 150, and $250 \mathrm{~s}$. 


\begin{tabular}{|c|c|c|c|c|c|c|c|c|c|c|c|c|c|c|c|c|c|}
\hline \multirow{3}{*}{9} & & 18 & & 26 & & 12 & 7 & 8 & 7 & 8 & & & 6 & & & \multicolumn{2}{|c|}{$6 \Rightarrow 7$} \\
\hline & $40^{2}$ & 28 & 29 & 24 & 27 & & 24 & 20 & 35 & 24 & 80 & 18 & 18 & 18 & 19 & 30 & \\
\hline & & 52 & 69 & 40 & 46 & 58 & 114 & 45 & 27 & 37 & 76 & 77 & 32 & 46 & 34 & 50 & \\
\hline 64 & & 28 & 34 & 44 & 76 & 64 & 65 & 26 & 13 & 27 & 35 & 51 & 46 & 35 & 49 & 63 & \\
\hline 34 & 39 & 23 & 19 & 36 & 59 & 49 & 62. & 48 & 33 & 37 & 24 & 19 & 43 & 68 & $4 \frac{3}{7}$ & & \\
\hline 20 & 395 & 34 & 40 & 40 & 41 & 49 & 81 & 142 & 62 & 31 & 28 & 32 & 40 & 8) & 60 & 54 & 27 \\
\hline 18 & 29 & 56 & 54 & $4 Q_{2}$ & 47 & 41 & 47 & $8 x^{3}$ & 100 & 65 & 53 & 51 & 44 & 315 & 51 & 66 & 32 \\
\hline 31 & 31 & 33 & 23 & 22 & 21 & 17 & 33 & 49 & 48 & 40 & 36 & 32 & 26 & 26 & -34 & $36^{2}$ & 27 \\
\hline 9 & 9 & 8 & 9 & 8 & 9 & 11 & 10 & 11 & 13 & 16 & 20 & 20 & 22 & 22 & 19 & 10 & 7 \\
\hline
\end{tabular}

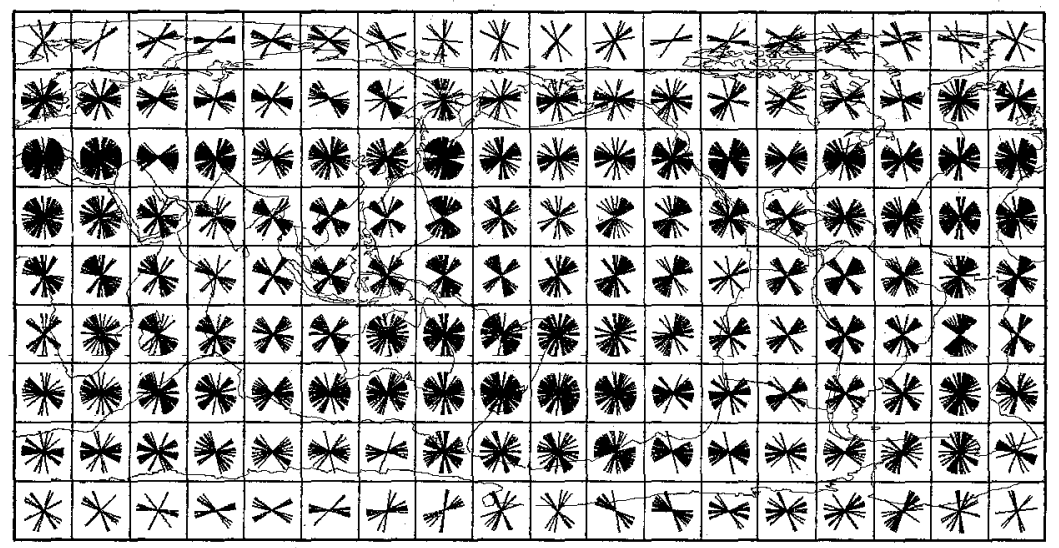

Fig. 2. Same as Figure 1 except that this is for Love wave at $200 \mathrm{~s}$.

can cause severe multipathing effects [e.g., Sobel and von Seggern, 1978].

Thus we face a problem of the lack of reasonable criterion for the cutoff level $p$. This situation does not change for Love waves or for the cases where azimuthal dependence is incorporated. A similar situation for the geomagnetic problem was reported by Whaler and Gubbins [1981]. For comparison, the case of Love wave heterogeneity inversion with $l \mathrm{max}=10$ is shown in Figure 4.

\section{Effect of the Level of Eigenvalue Cutoff $p$}

With no obvious criterion for selection of $p$, we can ask the questions (1) what are the effects of $p$ on the phase velocity variation map, and (2) what are the robust features in the map. Figure 5 shows the Rayleigh wave heterogeneity inversion at $200 \mathrm{~s}$ with $\operatorname{lmax}=10$. From top to bottom, $p$ is $71,81,91$, and 101. Contours are drawn at each $0.5 \%$ interval. Striped regions are positive (faster phase velocity) region, while the patterned regions are negative (slower). Zero lines (average velocity) are drawn thicker than other contours.

Three features we can see from Figure 5 are as follows:

1. The locations of peaks and troughs do not change much as $p$ is changed. Note, for example, the troughs (slower regions) in northeastern Africa, Tibet, near Fiji and New Zealand, and the East Pacific Rise to California and the peaks (faster regions) in the western Pacific, Australia, northeastern Pacific, and western Africa to northwestern part of Asia. These features seem to be quite robust.
2. Although the locations are robust, the total variation increases as $p$ is increased.

3. The shape of the zero contour changes, as $p$ is changed.

The same conclusions can be reached from Figure 6, which shows the results of Love wave heterogeneity inversions at 200 $\mathrm{s}$ with $l \max =10$. In this figure, $p$ is $47,53,59$, and 71 from top to bottom.

\section{Effect of Windowing}

Once the spherical harmonic coefficients are obtained, some kind of window should be applied to these coefficients in order to avoid the ringing phenomena associated with truncation at Imax. Effects of windowing are shown in Figure 7. The case of Love waves at $200 \mathrm{~s}$ with $l \mathrm{max}=10$ and $p=53$ is used in Figure 7.

The top part of Figure 7 corresponds to the Hamming window, i.e.,

$$
w(l)=0.54+0.46 \cos \left(\frac{\pi}{l \max } l\right)
$$

The same $w(l)$ is applied to all coefficients within the same angular order 1 .

The other three figures correspond to the windowing by the function

$$
w(l)=\cos \left(\frac{\pi}{2} \frac{l}{l \max +n}\right)
$$



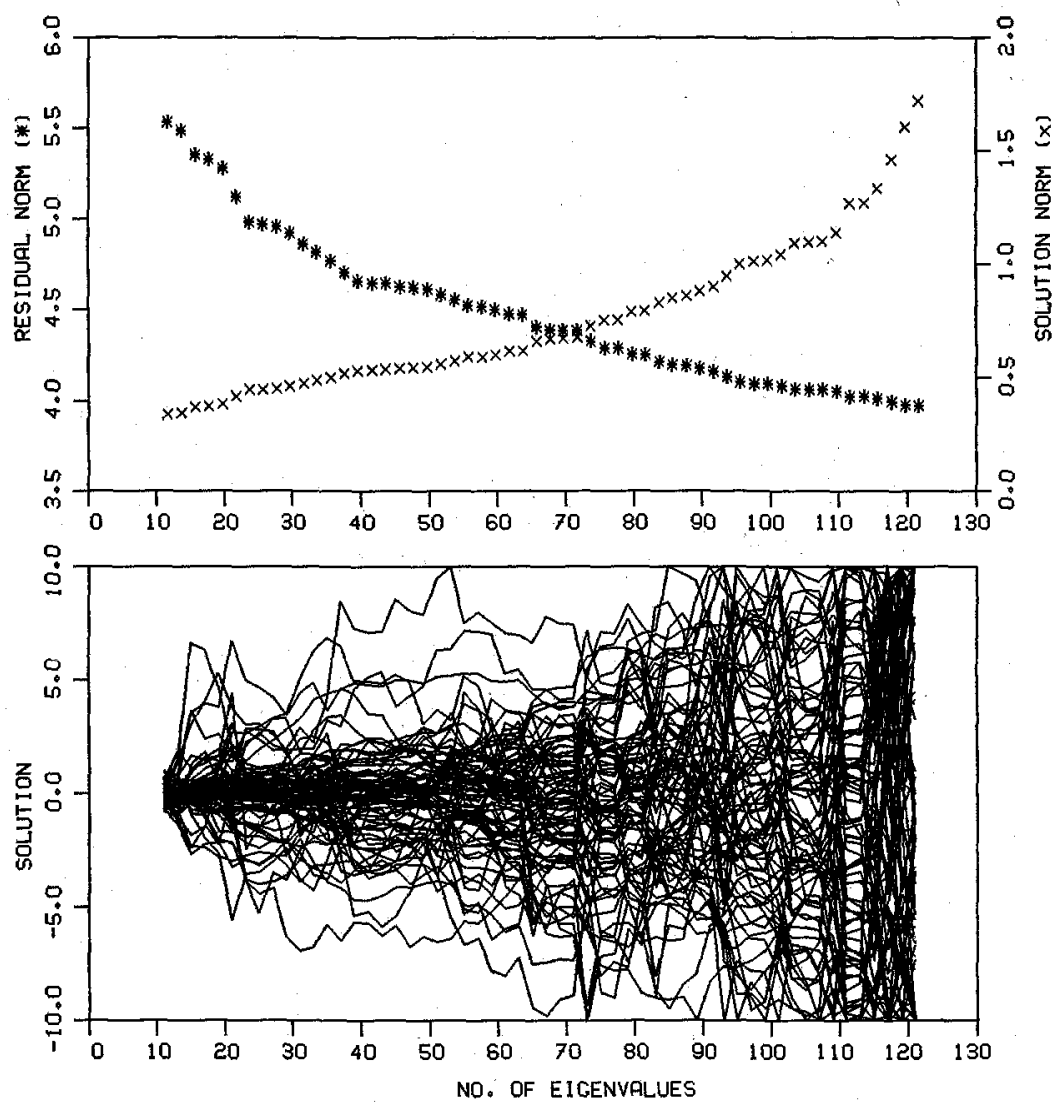

Fig. 3. Solution behavior of heterogeneity inversion with $l \mathrm{max}=10$. This is for Rayleigh waves at $200 \mathrm{~s}$. (Top) The residual norm (stars) and the solution norm (crosses) as a function of the number of eigenvalues kept $(p)$ in the solution. They are plotted from 11 to 121 at an interval of 2. (Bottom) The behavior of the solutions. Their behavior is wild for $p>90$ and especially so for $113 \leq p \leq 121$.
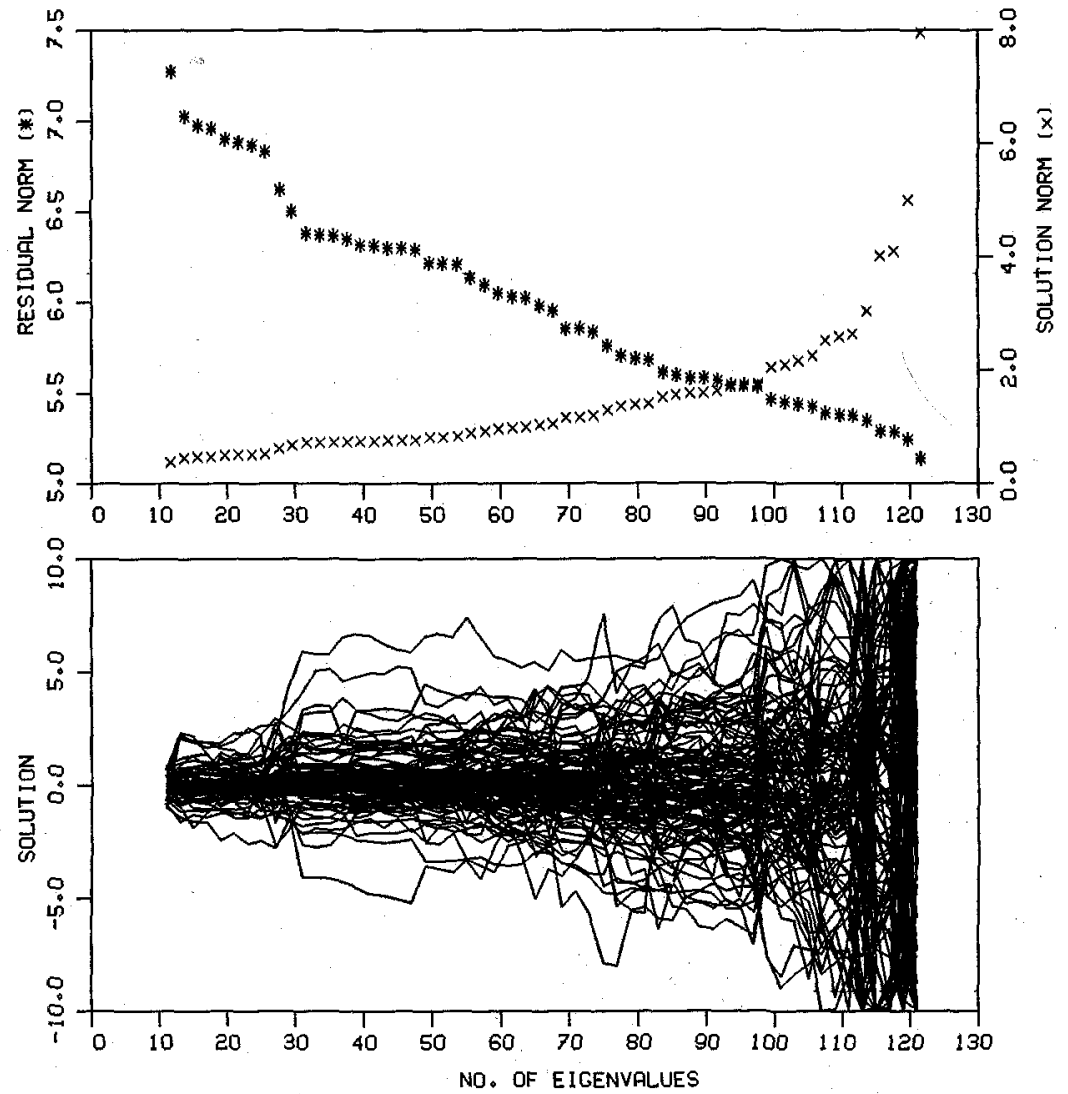

Fig. 4. Same as Figure 3 except for Love wave at $200 \mathrm{~s}$. 

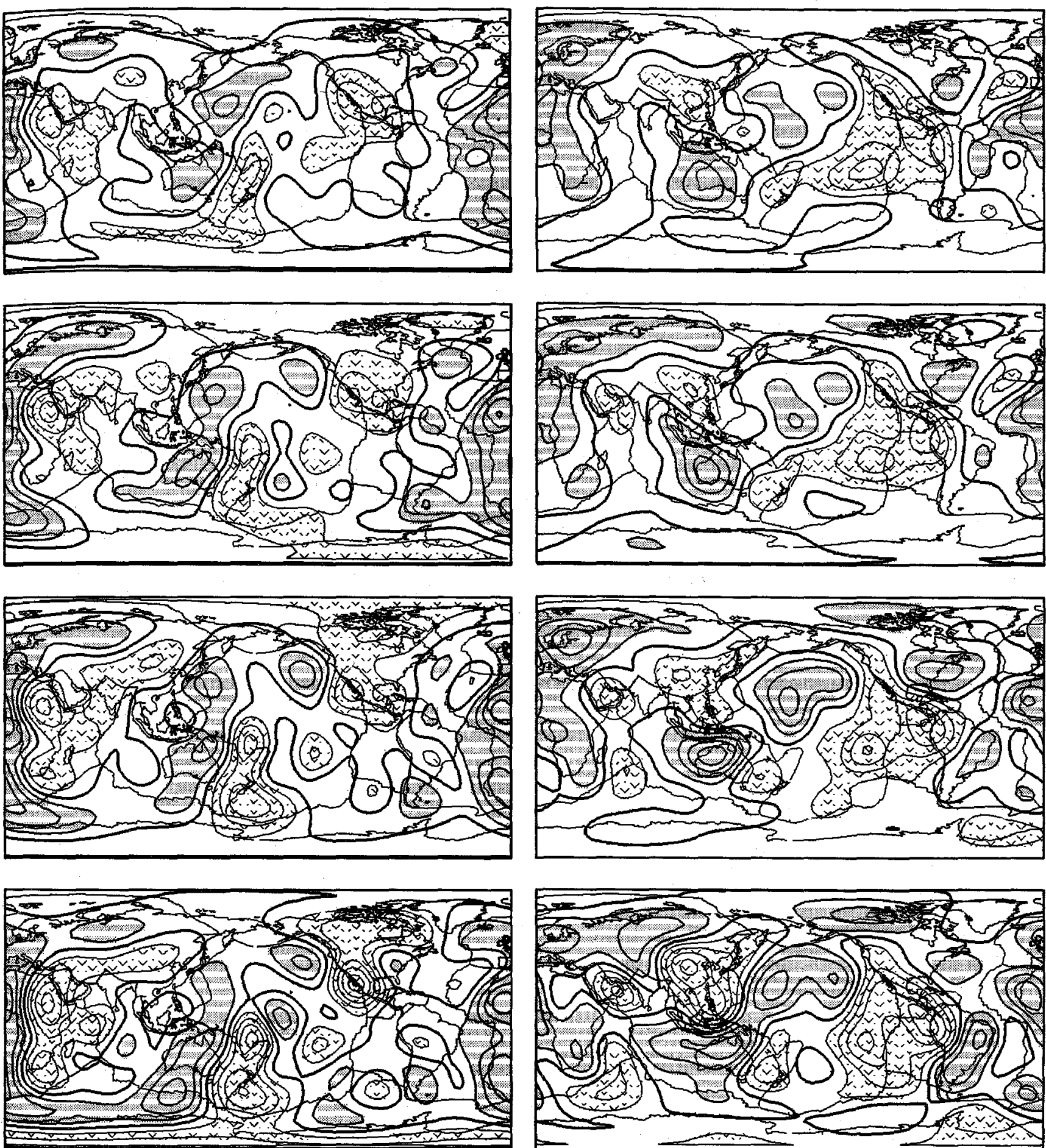

Fig. 5. Effect of the level of eigenvalue cutoff $p$. This is for Rayleigh wave heterogentiy inversion with $l \max =10$ at $200 \mathrm{~s}$. From top to bottom, $p$ is $71,81,91$, and 101 . Locations of peaks and troughs of phase velocities do not change much, while the maximum values increase as $p$ is increased. Contour interval for this and all other maps is $0.5 \%$.

The parameter $n$ is 1,2 , and 4 for the second, third, and fourth figure from top. The effect of changing $n$ is similar to the effect of changing the level of cutoff $p$, i.e., the locations of peaks and troughs do not change much but the zero contour changes as different windows are applied. As higher $l$ coefficients become less suppressed, more details emerge. For example, a positive peak in the central Pacific in the top three figures is broken up

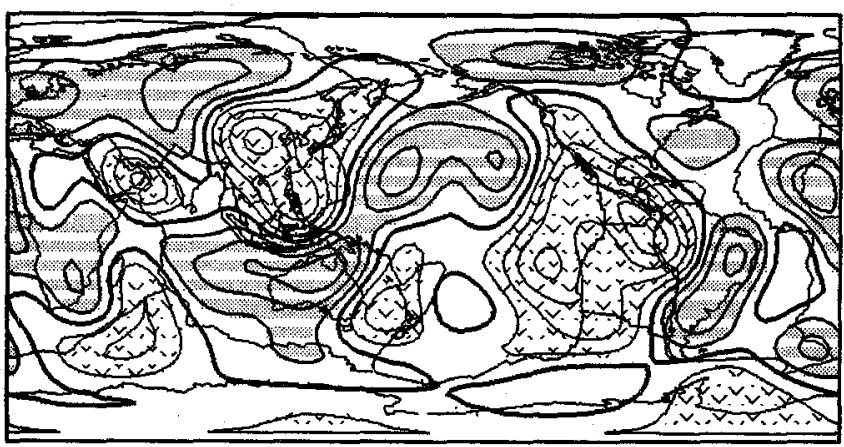

Fig. 6. Same as Figure 5 except for Love waves. From top to bottom, $p$ is $47,53,59$, and 71 .

into two peaks in the bottom figure. This is done, of course, at the risk of introducing spurious ringing in the map. We use the window of the bottom figure for all other figures in this paper unless otherwise noted.

\section{RESUlts AND DisCUSSION BY THE SPHERICAL}

\section{HARMONIC APPROACH}

In this section we show the results of inversions with and without the azimuthally dependent terms. In all cases, we set the eigenvalue cutoff level $p$, such that the solution norm does 

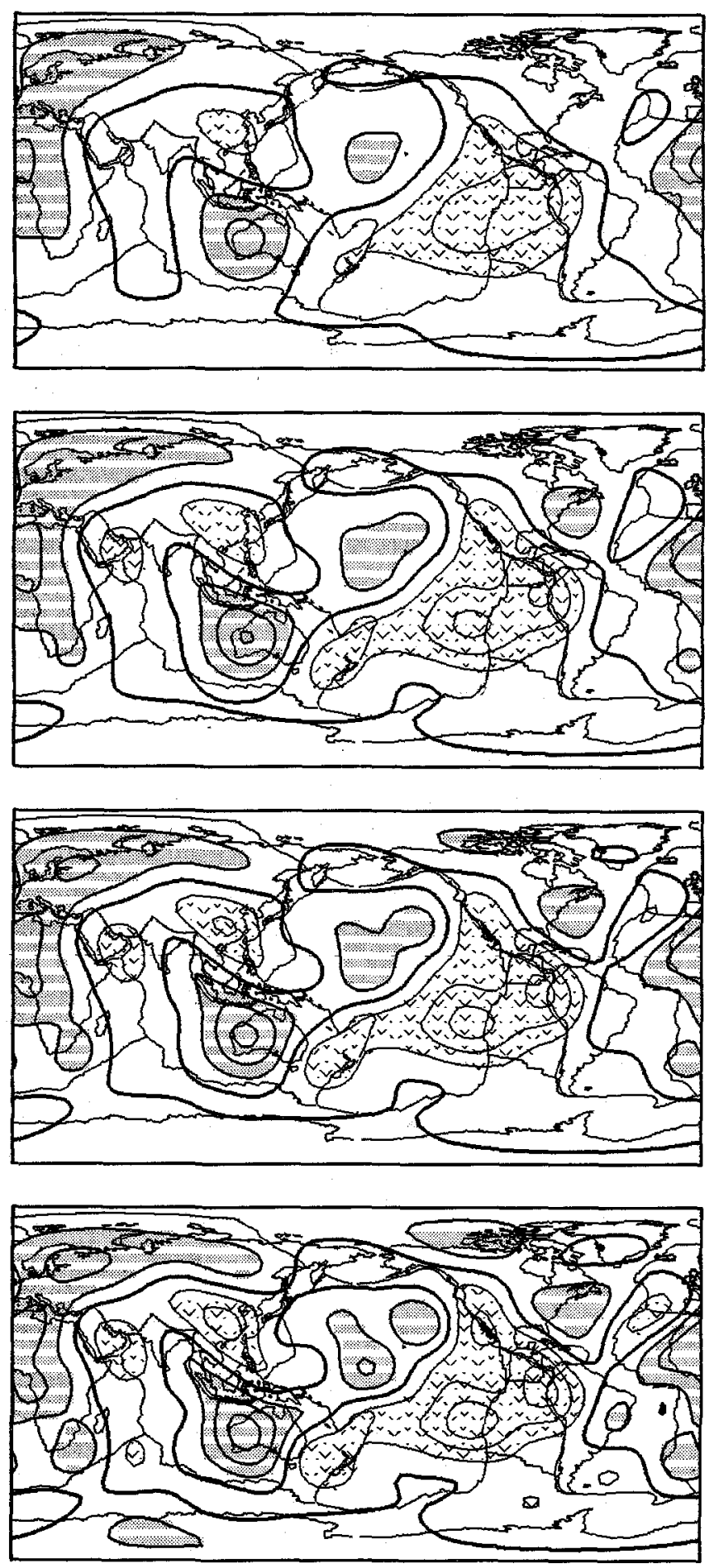

Fig. 7. Effect of windowing. The top figure is the result of Hamming window (equation (6)) and the rest are the results of windowing by the function in (7). The parameter $n$ is 1,2 , and 4 for the second, third, and fourth figure. The window with $n=4$ is used for all other figures in this paper. not exceed 0.008 . This has the effect of setting the maximum value of the phase velocity variations at about $2-2.5 \%$. As discussed in the last section, maximum values are not robust features of a solution, but locations of peaks and troughs are.

\section{Results for Heterogeneity Inversion}

We present the results for $l \max =10$ in this paper. Azimuthal dependence is not yet taken into account. The total number of parameters is $(\operatorname{lmax}+1)^{2}=121$ in this case. The numbers of eigenvalues kept in the solution are given in Table 4. Larger $p$ means better resolution. Rayleigh waves have better resolution than Love waves and in both cases the best resolution is achieved at $200 \mathrm{~s}$.

Figure 8 shows the results for Love waves. The results for $100,150,200$, and $250 \mathrm{~s}$ are given from top to bottom. There are excellent correlations with surface tectonics. Generally, old oceans and shields have fast phase velocities, while ridge regions and marginal seas have slow velocities. Subduction zones are generally characterized by slow velocity, which is presumably the effect of slow velocity in the back arc regions.

There are gradual changes from 100 to $200 \mathrm{~s}$, but the map for $250 \mathrm{~s}$ is quite different. There is, for example, a slow peak south of Africa, which does not exist clearly in the top three figures. Also, a fast peak occurs in the western Pacific and the fast peak in the northeastern Pacific disappeared. Moreover, the locations of the peaks have shifted. There is a possibility that this is due to the relatively poor fit of the model to the data. As shown in Table 5, the total variance reduction (VR) at $250 \mathrm{~s}$ is not as good as VR at other periods. VR is calculated by $\mathrm{VR}=\left(\sigma_{0}{ }^{2}-\sigma^{2}\right) / \sigma_{0}{ }^{2}$, where $\sigma_{0}{ }^{2}$ is the variance for the spherically symmetric earth and $\sigma^{2}$ is the variance for the aspherical model obtained by inversion. However, we do not think this is likely to be the cause for this case, since comparable difference of VR exists between 150 and $200 \mathrm{~s}$ without much difference in the locations of peaks. It is more likely to be caused by heterogeneity in deeper regions of the upper mantle, which is not correlated with shallower features.

The results for Rayleigh waves are given in Figure 9. There are quite a few similarities with the Love wave results. For example, slow velocities in the Red Sea-Gulf of Aden region to the triple junction in the south Indian ocean, Tibet, East Pacific Rise to California and Tasman Sea to New Zealand. Australia and the eastern Atlantic are fast in both sets of maps.

At the same time there exist some differences. The fast regions in the western Pacific are shifted somewhat. The Canadian shield is fast for Love waves and slow for long-period Rayleigh waves. Ridges are generally slow for Love waves, but they are not so evident on Rayleigh wave maps. Some ridge segments are, in fact, fast for Rayleigh waves. Subduction zones are characterized by slow velocity for Love waves, but some are fast for Rayleigh waves at long periods.

In terms of path coverage, Rayleigh waves have much better coverage than Love waves (Figures 1 and 2). Nakanishi and

TABLE 4. The Level of Eigenvalue Cutoff $p$ for Each Case of Inversion

\begin{tabular}{rcccccccccc}
\hline & & \multicolumn{4}{c}{ Love } & \multicolumn{4}{c}{ Rayleigh } \\
\cline { 3 - 7 } \cline { 5 - 9 } Imax & Anisotropy & $100 \mathrm{~s}$ & $150 \mathrm{~s}$ & $200 \mathrm{~s}$ & $250 \mathrm{~s}$ & & $100 \mathrm{~s}$ & $150 \mathrm{~s}$ & $200 \mathrm{~s}$ & $250 \mathrm{~s}$ \\
\hline 10 & & 43 & 53 & 54 & 53 & 49 & 65 & 82 & 74 \\
4 & $2 \Psi$ & 29 & 30 & 28 & 36 & 35 & 42 & 52 & 49 \\
4 & $2 \Psi+4 \Psi$ & 34 & 37 & 37 & 43 & 47 & 56 & 63 & 62 \\
6 & $2 \Psi$ & 40 & 40 & 43 & 46 & 52 & 55 & 63 & 63 \\
\hline
\end{tabular}



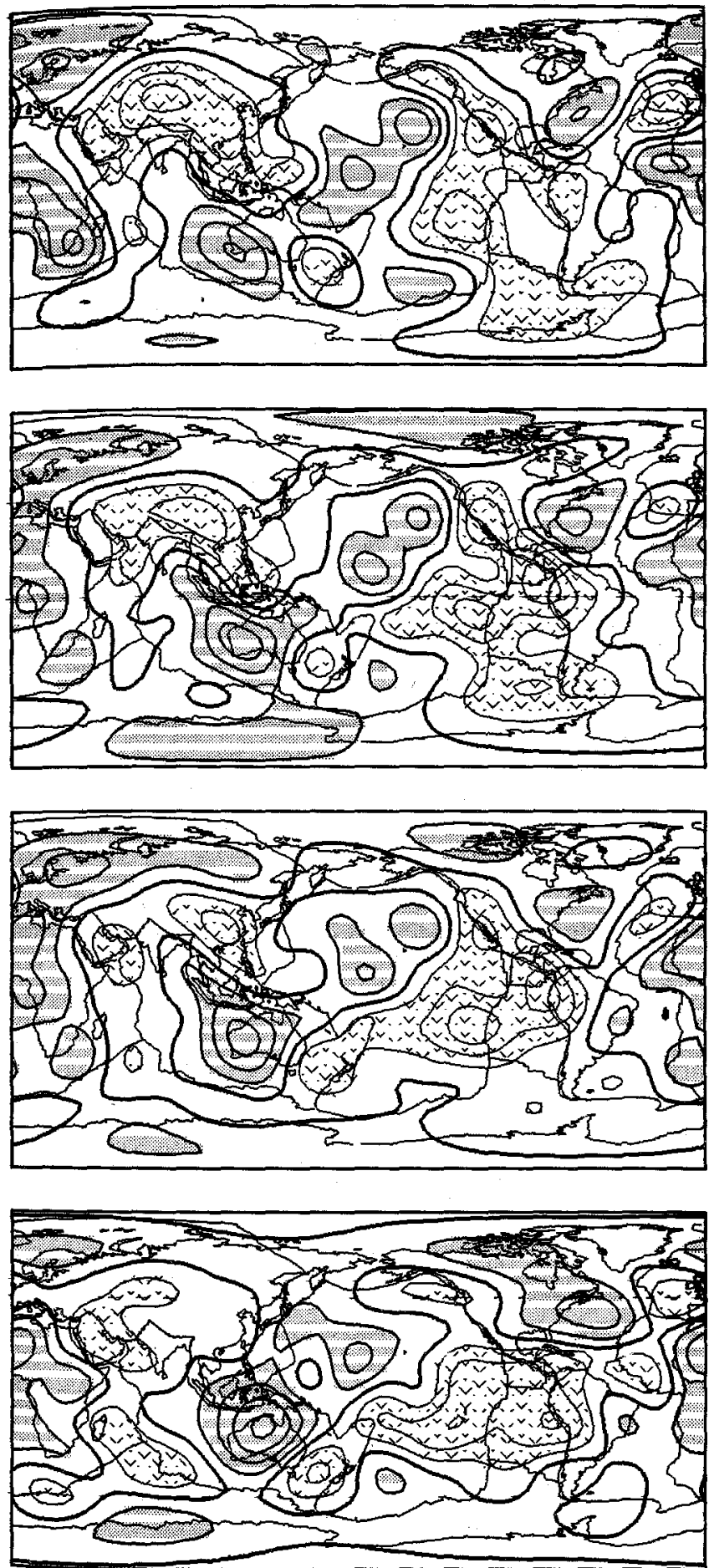

Fig. 8. Results of Love wave heterogeneity inversion with $l \max =10$. The results at $100,150,200$, and $250 \mathrm{~s}$ are shown from top to bottom.

Anderson [1984] discussed the problem of source depth errors on Rayleigh wave initial phase. However, VR of Rayleigh wave is larger than that of Love waves (Table 5). Thus it is more likely that the data should be explained by differences in penetration depth or transverse isotropy. Differences in subduction zones, slow for Love waves and fast for Rayleigh waves, are probably due to the difference in penetration depth, since fast velocity at depth is consistent with the subduction of cold, fast lithosphere. Love wave-Rayleigh wave differences at trenches and ridges can also be caused by transverse isotropy.
TABLE 5. Variance Reduction

\begin{tabular}{lccccc}
\hline & & $100 \mathrm{~s}$ & $150 \mathrm{~s}$ & $200 \mathrm{~s}$ & $250 \mathrm{~s}$ \\
& $l \max$ & VR, \% & VR, \% & VR, \% & VR, \% \\
\hline Love & 10 & 40.5 & 39.3 & 44.1 & 36.7 \\
Rayleigh & 10 & 45.8 & 64.9 & 66.6 & 54.5 \\
\hline
\end{tabular}

This is because $S H$ may be less than $S V$ due, presumably, to ascending and descending flow in the mantle [Anderson and Regan, 1983], although, in general, $S H>S V$ in the shallow mantle [Anderson and Dziewonski, 1982].
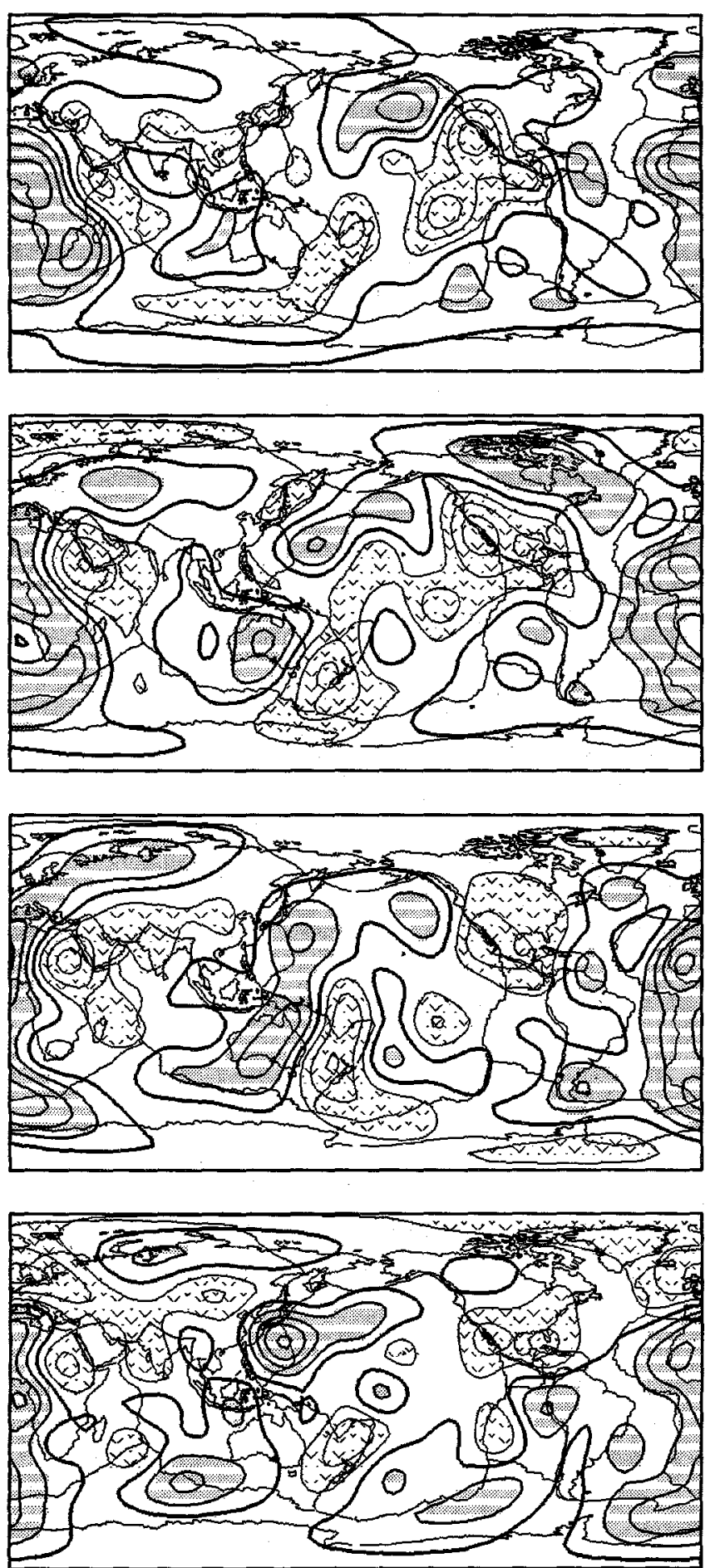

Fig. 9. Same as Figure 8 except for Rayleigh waves. 
TABLE 6. Spherical Harmonic Coefficients for Love and Rayleigh Waves at 150 and $200 \mathrm{~s}$

\begin{tabular}{|c|c|c|c|c|c|c|c|c|c|c|c|}
\hline \multirow{3}{*}{1} & \multirow[b]{3}{*}{$\mathrm{m}$} & \multicolumn{2}{|c|}{ Love } & \multicolumn{2}{|c|}{ Rayleigh } & \multirow[b]{2}{*}{1} & \multirow[b]{2}{*}{$\mathrm{m}$} & \multicolumn{2}{|c|}{ Love } & \multicolumn{2}{|c|}{ Rayleigh } \\
\hline & & & & & & & & $150 \mathrm{~s}$ & $200 \mathrm{~s}$ & $150 \mathrm{~s}$ & $200 \mathrm{~s}$ \\
\hline & & $150 \mathrm{~s}$ & $200 \mathrm{~s}$ & $150 \mathrm{~s}$ & $200 \mathrm{~s}$ & 8 & $4 \mathrm{C}$ & 0.00008 & -0.00013 & 0.00049 & -0.00018 \\
\hline 0 & $0 \mathrm{C}$ & 0.00013 & 0.00040 & 0.00117 & 0.00006 & 8 & $4 \mathrm{~S}$ & -0.00029 & 0.00005 & 0.00068 & 0.00067 \\
\hline 1 & $0 \mathrm{C}$ & 0.00009 & 0.00088 & -0.00037 & -0.00046 & 8 & $5 \mathrm{C}$ & 0.00062 & 0.00031 & -0.00054 & -0.00058 \\
\hline 1 & $1 \mathrm{C}$ & 0.00087 & 0.00157 & 0.00273 & 0.00129 & 8 & $5 \mathrm{~S}$ & 0.00024 & 0.00038 & -0.00033 & 0.00036 \\
\hline 1 & $1 \mathrm{~S}$ & 0.00181 & 0.00206 & 0.00006 & 0.00031 & 8 & $6 \mathrm{C}$ & 0.00027 & -0.00022 & 0.00007 & -0.00001 \\
\hline 2 & $0 \mathrm{C}$ & 0.00074 & 0.00071 & 0.00046 & 0.00016 & 8 & $6 \mathrm{~S}$ & -0.00014 & -0.00019 & 0.00007 & 0.00024 \\
\hline 2 & $1 \mathrm{C}$ & 0.00045 & -0.00011 & -0.00111 & -0.00115 & 8 & $7 \mathrm{C}$ & -0.00025 & -0.00016 & 0.00138 & 0.00078 \\
\hline 2 & $1 \mathrm{~S}$ & -0.00241 & -0.00149 & 0.00028 & 0.00023 & 8 & $7 \mathrm{~S}$ & -0.00052 & -0.00044 & 0.00129 & 0.00081 \\
\hline 2 & $2 \mathrm{C}$ & 0.00279 & 0.00262 & 0.00171 & 0.00194 & 8 & $8 \mathrm{C}$ & -0.00005 & 0.00008 & -0.00028 & -0.00028 \\
\hline 2 & $2 \mathrm{~S}$ & -0.00092 & -0.00133 & -0.00211 & -0.00258 & 8 & $8 \mathrm{~S}$ & 0.00007 & -0.00045 & 0.00011 & 0,00038 \\
\hline 3 & $0 \mathrm{C}$ & 0.00053 & 0.00019 & 0.00112 & 0.00042 & 9 & $0 \mathrm{C}$ & 0.00027 & -0.00017 & 0.00012 & -0.00001 \\
\hline 3 & $1 \mathrm{C}$ & -0.00046 & -0.00006 & 0.00043 & 0.00089 & 9 & $1 \mathrm{C}$ & -0.00067 & -0.00043 & 0.00009 & -0.00075 \\
\hline 3 & $1 \mathrm{~S}$ & -0.00026 & -0.00061 & -0.00060 & 0.00030 & 9 & $1 \mathrm{~s}$ & -0.00030 & -0.00039 & 0.00020 & 0.00032 \\
\hline 3 & $2 \mathrm{C}$ & 0.00072 & 0.00093 & 0.00104 & 0.00166 & 9 & $2 \mathrm{C}$ & 0.00041 & 0.00019 & 0.00005 & -0.00017 \\
\hline 3 & $2 S$ & 0.00041 & 0.00103 & -0.00053 & 0.00002 & 9 & $2 \mathrm{~S}$ & 0.00041 & 0.00050 & -0.00017 & 0.00011 \\
\hline 3 & $3 \mathrm{C}$ & 0.00001 & 0.00046 & 0.00120 & 0.00181 & 9 & $3 \mathrm{C}$ & -0.00007 & 0.00012 & 0.00003 & -0.00021 \\
\hline 3 & $3 \mathrm{~S}$ & -0.00083 & -0.00056 & -0.00092 & -0.00099 & 9 & $3 \mathrm{~S}$ & 0.00024 & -0.00008 & 0.00021 & 0.00077 \\
\hline 4 & $0 \mathrm{C}$ & 0.00035 & 0.00025 & -0.00084 & -0.00065 & 9 & $4 \mathrm{C}$ & -0.00025 & 0.00001 & -0.00031 & -0.00024 \\
\hline 4 & $1 \mathrm{C}$ & 0.00070 & 0.00068 & -0.00043 & -0.00034 & 9 & $4 \mathrm{~S}$ & -0.00024 & 0.00032 & -0.00049 & -0.00036 \\
\hline 4 & $1 \mathrm{~S}$ & 0.00111 & 0.00125 & -0.00024 & 0.00031 & 9 & $5 \mathrm{C}$ & -0.00024 & -0.00067 & -0.00052 & -0.00021 \\
\hline 4 & $2 \mathrm{C}$ & -0.00012 & -0.00074 & -0.00061 & 0.00036 & 9 & $5 \mathrm{~S}$ & 0.00038 & -0.00006 & -0.00030 & 0.00082 \\
\hline 4 & $2 \mathrm{~S}$ & 0.00011 & 0.00068 & 0.00097 & 0.00059 & 9 & $6 \mathrm{C}$ & -0.00020 & 0.00000 & -0.00026 & -0.00156 \\
\hline 4 & $3 \mathrm{C}$ & -0.00112 & -0.00115 & -0.00137 & -0.00117 & 9 & $6 \mathrm{~S}$ & -0.00011 & 0.00040 & 0.00083 & 0.00066 \\
\hline 4 & $3 \mathrm{~S}$ & 0.00001 & 0.00010 & -0.00085 & -0.00018 & 9 & $7 \mathrm{C}$ & -0.00082 & -0.00037 & 0.00016 & 0.00026 \\
\hline 4 & $4 \mathrm{C}$ & 0.00065 & 0.00057 & 0.00072 & 0.00021 & 9 & $7 \mathrm{~S}$ & 0.00046 & -0.00003 & -0.00046 & -0.00029 \\
\hline 4 & $4 \mathrm{~S}$ & 0.00138 & 0.00143 & 0.00019 & -0.00023 & 9 & $8 \mathrm{C}$ & 0.00018 & 0.00006 & 0.00011 & 0.00066 \\
\hline 5 & $O \mathrm{C}$ & 0.00021 & 0.00004 & -0.00090 & 0.00038 & 9 & $8 \mathrm{~S}$ & 0.00001 & 0.00018 & -0.00032 & -0.00039 \\
\hline 5 & $1 \mathrm{C}$ & 0.00004 & -0.00002 & 0.00042 & 0.00094 & 9 & $9 \mathrm{C}$ & -0.00028 & -0.00024 & 0.00006 & 0.00001 \\
\hline 5 & $1 \mathrm{~S}$ & 0.00134 & 0.00094 & -0.00075 & 0.00048 & 9 & $9 \mathrm{~S}$ & 0.00005 & 0.00006 & 0.00008 & -0.00058 \\
\hline 5 & $2 \mathrm{C}$ & -0.00086 & -0.00090 & -0.00122 & -0.00026 & 10 & $0 \mathrm{C}$ & -0.00009 & -0.00017 & 0.00043 & -0.00016 \\
\hline 5 & $2 \mathrm{~S}$ & -0.00067 & -0.00084 & 0.00040 & 0.00051 & 10 & $1 \mathrm{C}$ & -0.00090 & -0.00013 & -0.00015 & -0.00008 \\
\hline 5 & $3 \mathrm{C}$ & 0.00010 & 0.00005 & -0.00045 & 0.00026 & 10 & $1 \mathrm{~S}$ & -0.00081 & -0.00059 & -0.00106 & -0.00062 \\
\hline 5 & $3 \mathrm{~S}$ & 0.00058 & 0.00102 & 0.00007 & -0.00010 & 10 & $2 \mathrm{C}$ & -0.00028 & -0.00004 & 0.00029 & -0.00047 \\
\hline 5 & $4 \mathrm{C}$ & 0.00040 & 0.00114 & 0.00083 & 0.00016 & 10 & $2 \mathrm{~S}$ & 0.00088 & 0.00097 & 0.00057 & 0.00096 \\
\hline 5 & $4 \mathrm{~S}$ & 0.00035 & -0.00010 & -0.00058 & -0.00037 & 10 & $3 \mathrm{C}$ & 0.00049 & 0.00098 & 0.00020 & -0.00016 \\
\hline 5 & $5 \mathrm{C}$ & -0.00021 & -0.00013 & 0.00043 & 0.00210 & 10 & $3 \mathrm{~S}$ & 0.00017 & 0.00020 & 0.00107 & -0.00020 \\
\hline 5 & $5 \mathrm{~S}$ & 0.00051 & 0.00046 & -0.00019 & -0.00062 & 10 & $4 \mathrm{C}$ & -0.00039 & -0.00041 & -0.00027 & 0.00023 \\
\hline 6 & $0 \mathrm{C}$ & 0.00072 & 0.00023 & -0.00040 & -0.00089 & 10 & $4 \mathrm{~S}$ & -0.00085 & -0.00056 & -0.00053 & -0.00051 \\
\hline 6 & $1 \mathrm{C}$ & 0.00029 & 0.00019 & 0.00016 & 0.00013 & 10 & $5 \mathrm{C}$ & -0.00117 & -0.00117 & -0.00024 & -0.00065 \\
\hline 6 & $1 \mathrm{~S}$ & -0.00026 & -0.00049 & -0.00052 & 0.00015 & 10 & $5 \mathrm{~S}$ & 0.00006 & -0.00047 & -0.00076 & -0.00062 \\
\hline 6 & $2 \mathrm{C}$ & 0.00044 & -0.00014 & -0.00071 & -0.00034 & 10 & $6 \mathrm{C}$ & -0.00038 & 0.00020 & 0.00104 & 0.00002 \\
\hline 6 & $2 \mathrm{~S}$ & 0.00065 & 0.00086 & 0.00056 & 0.00054 & 10 & $6 \mathrm{~S}$ & 0.00089 & 0.00037 & 0.00032 & 0.00076 \\
\hline 6 & $3 \mathrm{C}$ & 0.00016 & -0.00032 & -0.00159 & -0.00061 & 10 & $7 \mathrm{C}$ & 0.00090 & 0.00071 & 0.00065 & 0.00015 \\
\hline 6 & $3 \mathrm{~S}$ & 0.00095 & 0.00109 & 0.00069 & 0.00050 & 10 & $7 \mathrm{~S}$ & 0.00178 & 0.00157 & 0.00137 & 0.00123 \\
\hline 6 & $4 \mathrm{C}$ & -0.00092 & -0.00035 & -0.00061 & -0.00046 & 10 & $8 \mathrm{C}$ & 0.00123 & 0.00069 & 0.00028 & 0.00070 \\
\hline 6 & $4 \mathrm{~S}$ & 0.00170 & 0.00114 & 0.00116 & 0.00091 & 10 & $8 \mathrm{~S}$ & -0.00105 & -0.00097 & -0.00059 & -0.00029 \\
\hline 6 & $5 \mathrm{C}$ & 0.00100 & 0.00119 & 0.00114 & 0.00060 & 10 & $9 \mathrm{C}$ & $-0,00008$ & -0.00068 & -0.00091 & -0.00093 \\
\hline 6 & $5 \mathrm{~S}$ & 0.00053 & 0.00089 & 0.00113 & 0.00120 & 10 & $9 \mathrm{~S}$ & 0.00106 & 0.00058 & 0.00014 & 0.00019 \\
\hline 6 & $6 \mathrm{C}$ & -0.00006 & 0.00016 & -0.00081 & 0.00019 & 10 & $10 \mathrm{C}$ & 0.00061 & 0.00048 & -0.00064 & -0.00049 \\
\hline 6 & $6 \mathrm{~S}$ & 0.00039 & 0.00045 & 0.00046 & 0.00014 & 10 & $10 \mathrm{~S}$ & 0.00061 & 0.00032 & 0.00013 & 0.00050 \\
\hline
\end{tabular}

These numbers multiplied by phase velocities of Preliminary Reference Earth Model (PREM) give deviations from PREM.

Compared with previous studies by Nakanishi and Anderson $[1983,1984](l \max =6)$, the overall patterns are very similar; slow regions at northeast Africa, Tibet, Tasman Sea, and southwestern North America; triple junctions in the north Atlantic and south Indian Ocean; and fast regions at Australia, western Pacific, and the southern Atlantic. However, a few detailed features have emerged. Most notable is the breakup of the fast regions in the Pacific. This appears clearly for Love waves at 100,150 , and $200 \mathrm{~s}$ and Rayleigh waves at 150 and $200 \mathrm{~s}$. In terms of the numbers of data and the variance reductions (VR), the results at 150 and $200 \mathrm{~s}$ are most reliable (Table 5). Thus we believe that this apparent increase in resolution is real. We tabulate the coefficients for Rayleigh and Love waves at 150 and $200 \mathrm{~s}$ in Table 6. 


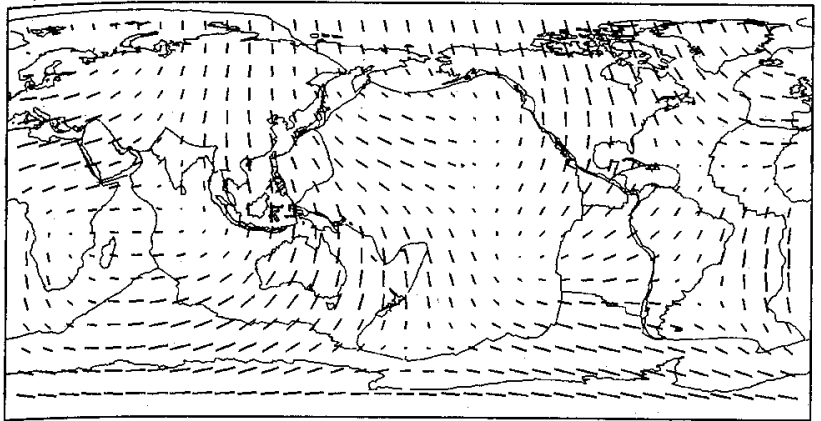

$l \max =6$ $l=1-3$

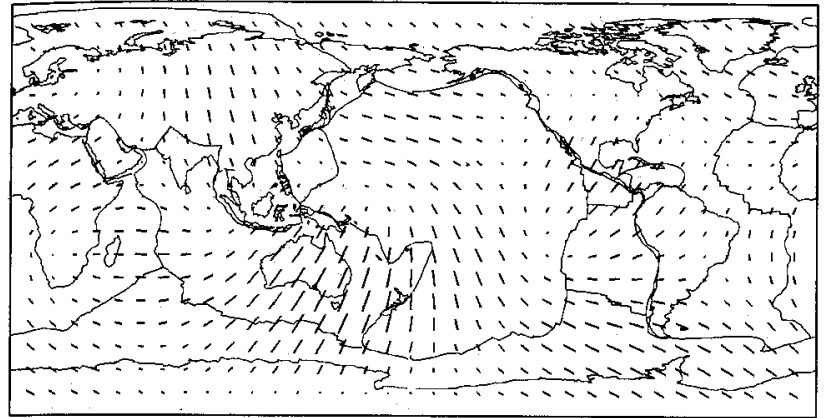

-1 per cent

Fig. 10. Results of inversion with azimuthally dependent terms for Rayleigh waves at $200 \mathrm{~s}$. The $2 \Psi$ terms as well as heterogeneity terms with $l \mathrm{max}=4$ (top) and $l \mathrm{max}=6$ (bottom) are inverted. Plotted in this figure are the fastest direction of phase velocity at each point. Length of lines specify velocity difference between the fastest direction and the slowest (perpendicular) direction. Results for $l=1-3$ are plotted.

\section{Results for Azimuthal Dependence}

When azimuthal dependence is included, the number of parameters increases considerably. For heterogeneity plus $2 \Psi$ azimuthal inversion there are 3 times as many parameters, and for heterogeneity plus $2 \Psi$ and $4 \Psi$ terms there are 5 times as many parameters. Thus $l$ max has to be decreased from 10 , and we discuss the results with $2 \Psi$ dependence in this paper.

We present the results of two cases in this section. In case 1 we take $\operatorname{lmax}=4$ both for heterogeneity and $2 \Psi$ azimuthal dependence. In case 2 we take $l \max =6$. The term $l \max =0$ is excluded from the azimuthally dependent terms. In Figure 10, the results for case 1 are shown in the upper figure and those for case 2 in the lower figure. The lines on the maps give the direction of fastest phase velocity at each point. The length of each line gives the difference of velocity between the fastest and the slowest velocity at a point. In Figure 10 we plot the results of $2 \Psi$ azimuthal dependence for $l=1-3$. For comparison, the results for $l=1-4$ are given in Figure 11. Although there exist some differences among these figures, similarities also exist. For example, NW-SE fastest direction in most of the Pacific Ocean and NNE-SSW fastest direction in western Australia persist in all figures. Generally, there seems to be a good correlation with plate motions, but care must be taken, since resolution analysis in the next section suggest that our data have resolving power for $2 \Psi$ dependence only for particular regions. At the same time, however, the analysis in the next section is done for each region and not for a particular spherical harmonic component in the solution. The data may have resolution for low angular order spherical harmonics like $l=1-4$, and this may be a reason we find similarities in Figures 10 and 11 .

There is a tradeoff between heterogeneity and anisotropy. Figures $12 a$ and $12 b$ show results for Love waves in case $2(l \max =6)$, which were obtained by inversion with and without the $2 \Psi$ azimuthally dependent terms, respectively. Periods are $100,150,200$, and $250 \mathrm{~s}$ from top to bottom. Comparisons of Figures $12 a$ and $12 b$ reveal that the locations of peaks and troughs are not so different, but definitely there exists tradeoff, since the maximum values in Figure $12 a$ are much smaller. This is also confirmed by the resolution analysis in the next section.

\section{The Backus-Gilbert Approach}

In the preceding sections we examined the results by cutoff levels of eigenvalues and variance reductions. Another method to analyze the reliability of results is the Backus-Gilbert method [Backus and Gilbert, 1967, 1968, 1970]. Since this method constructs the solution from the available data, the null space in the problem is suppressed from the outset, and resolution kernels give an unbiased estimate of the resolving power of the data.

\section{Heterogeneity}

First, we rewrite equation (1) as

$$
\frac{1}{v_{i}}=\frac{1}{\Delta_{S R}} \int_{S}^{R} \frac{d s}{V(\theta, \varphi, \Psi)}
$$

and expand the path into spherical harmonics so that each path has a finite width. We use spherical harmonics up to $1=m=10$ to do this. Rigorously, one must go to higher
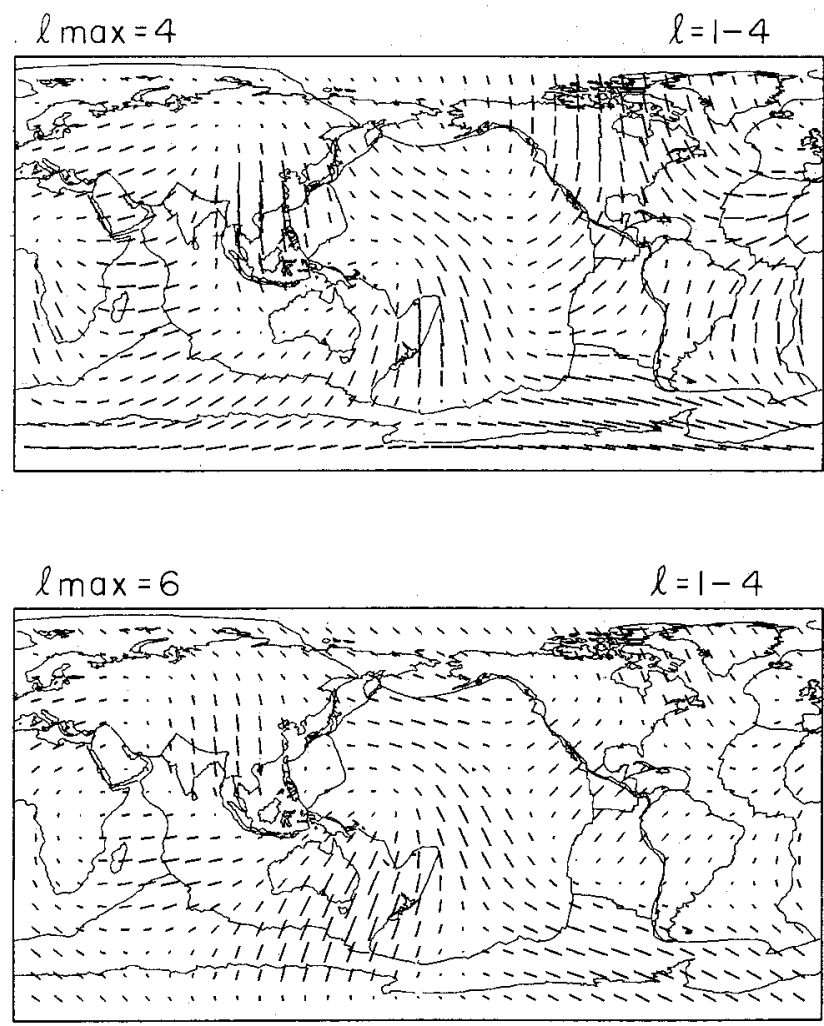

-1 per cent

Fig. 11. Same as Figure 10 except that $l=1-4$ are plotted. 

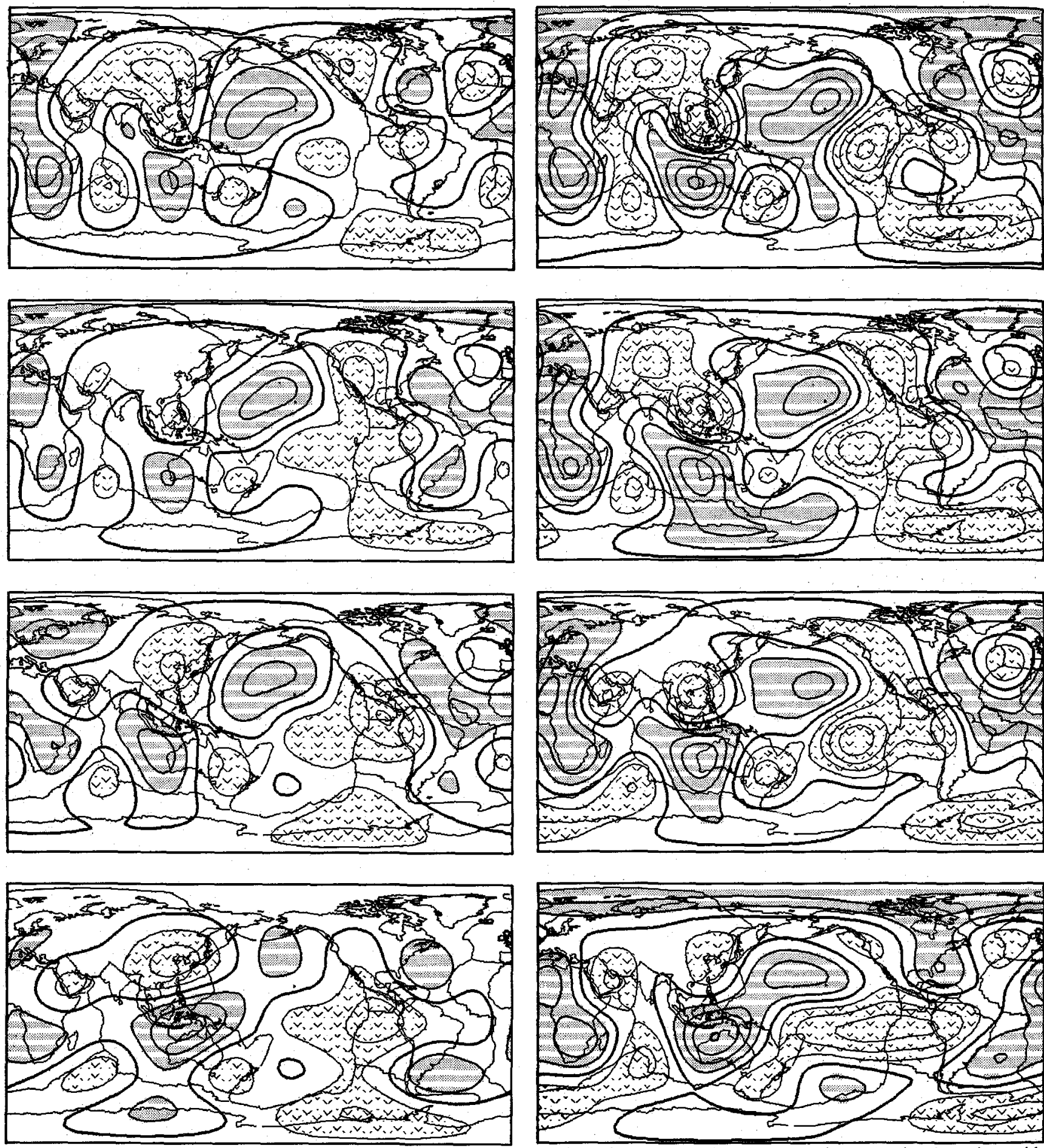

Fig. $12 a$. Phase velocity variation obtained by Love wave inversion with $2 \Psi$ azimuthally dependent terms. Azimuthal dependence obtained simultaneously is shown in Figure 10. In this and the next figures (Figure $12 b$ ), no window is applied.

angular orders to represent faithfully 100 - to 250 -s waves and also give different width for different periods. In this sense, the present approach is preliminary and contains some room for improvment.

We obtain

$$
s_{i}=\int d \Omega \cdot K_{i}(\theta, \varphi) S(\theta, \varphi)
$$

where $s_{i}$ is the ith datum in the slowness measurements, $K_{i}(\theta$, $l \max =6$. Locations of peaks and troughs are similar with Figure $12 a$, but their maximum values are much higher. This shows the existence of tradeoff in the inversion between heterogeneity and azimuthal dependence.

$\varphi)$ the appropriate kernel for the path, and $S(\theta, \varphi)$ the real slowness variation we could like to obtain.

We use the deltaness criterion. We take a linear combination of the data

$$
\bar{S}\left(\theta_{0}, \varphi_{0}\right) \equiv \sum_{i} \alpha_{i} s_{i}=\int d \Omega\left\{\sum_{i} \alpha_{i} K_{i}(\alpha, \varphi)\right\} S(\theta, \varphi)
$$

and take $\alpha_{i}$ such that $\Sigma_{i} \alpha_{i} k_{i}(\theta, \varphi)$ becomes as closely as 


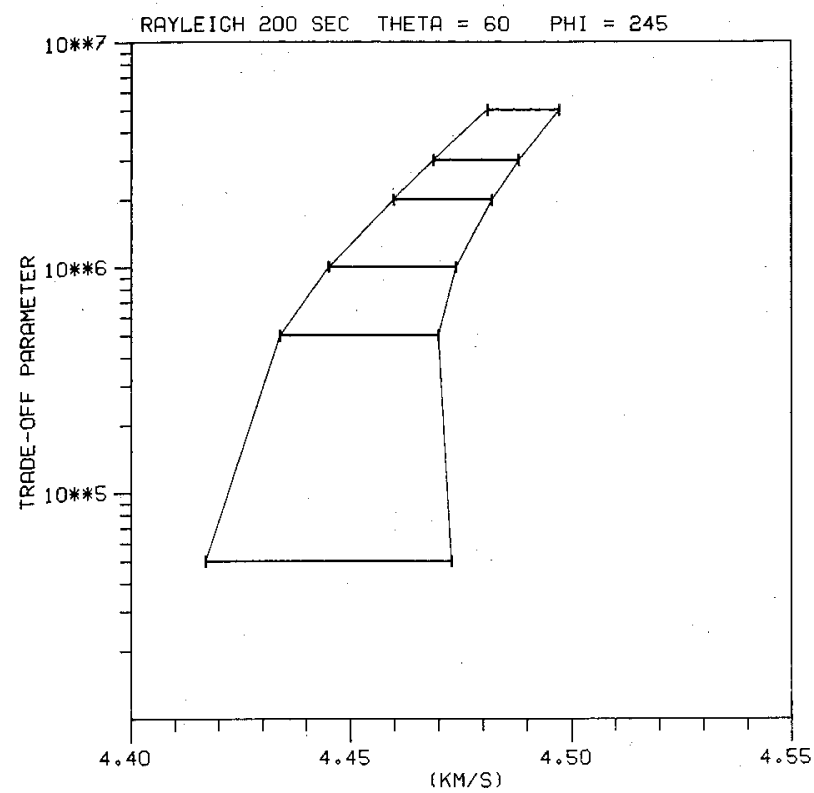

Fig. $13 a$

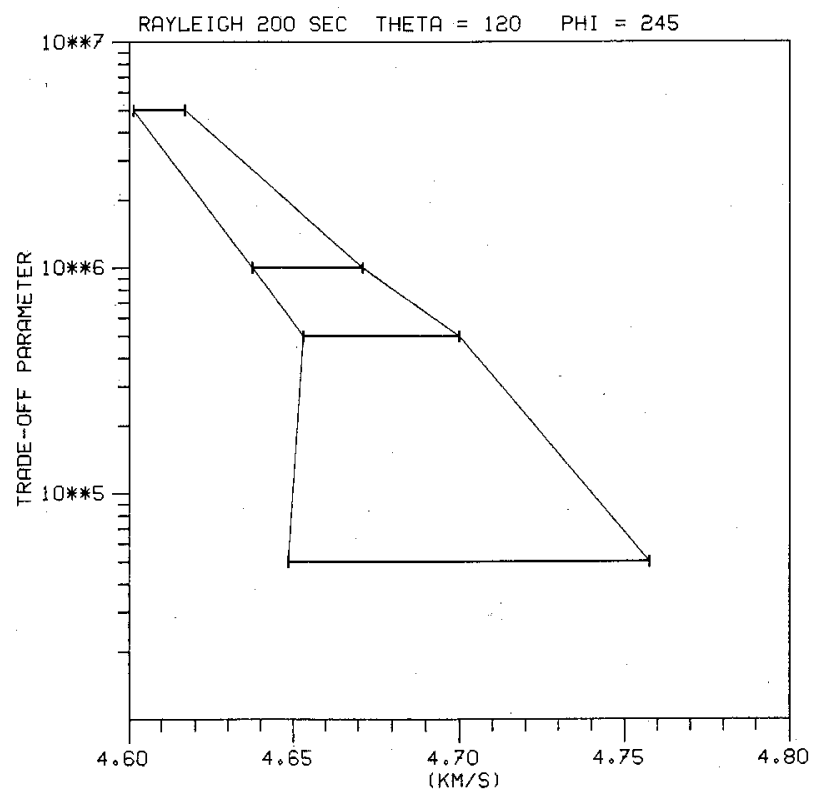

Fig. $13 b$

Fig. 13. Semi-log plots of tradeoff parameters versus estimated phase velocity bounds. They are Rayleigh waves at $200 \mathrm{~s}$ for locations near (a) California $\left(30^{\circ} \mathrm{N}, 115^{\circ} \mathrm{W}\right)$ and $(b)$ East Pacific Rise $\left(30^{\circ} \mathrm{S}\right.$, $\left.115^{\circ} \mathrm{W}\right)$

possible to $\delta\left(\varphi-\varphi_{0}\right) \delta\left(\cos \theta-\cos \theta_{0}\right)$. We determine $\alpha_{i}$ by minimizing the following formula $J$ :

$$
J=\alpha^{t} G \alpha+\tan \lambda \alpha^{t} V \alpha-2 \alpha^{t} K\left(\theta_{0}, \varphi_{0}\right)
$$

where

$$
\begin{gathered}
(G)_{i j}=\int d \Omega K_{i}(\theta, \varphi) K_{j}(\theta, \varphi) \\
K\left(\theta_{0}, \varphi_{0}\right)=\left(K_{1}\left(\theta_{0}, \varphi_{0}\right), K_{2}\left(\theta_{0}, \varphi_{0}\right), \cdots\right)^{t}
\end{gathered}
$$

$\tan \lambda$ is tradeoff parameter and $V$ is the covariance matrix of data.

Tradeoff parameter $\tan \lambda$ was determined from the following observations. Figure $13 a$ shows phase velocity bounds obtained for different tradeoff parameters. This is for a point near California $\left(30^{\circ} \mathrm{N}, 115^{\circ} \mathrm{W}\right)$, and the data are Rayleigh waves at $200 \mathrm{~s}$. Note that this is a semi-log plot. As tradeoff parameters are increased, velocity bounds become smaller, while kernels deviate away from the delta function. This is a well-known fact in inverse theory. We also notice a clear systematic deviation of velocity bounds for tradeoff parameters larger than about $5 \times 10^{5}$. The same trend is also observed at different locations. Figure $13 b$ is an example at a point near East Pacific Rise $\left(30^{\circ} \mathrm{S}, 115^{\circ} \mathrm{W}\right)$, which also shows systematic deviation of velocity bounds for $\tan \lambda$ larger than about 5 $\times 10^{5}$. This systematic deviation is mainly caused by antipodal contamination. Figure 14 shows kernels for $\tan \lambda=5$ $\times 10^{4}, 5 \times 10^{5}$, and $5 \times 10^{6}$ in the case of a point near the East Pacific Rise (Figure 13b). Peaks at the antipode are a little over $10 \%$ of the peak at the point we tried to construct a delta function $\left(30^{\circ} \mathrm{S}, 115^{\circ} \mathrm{W}\right)$ for $\tan \lambda=5 \times 10^{4}$ and about $20 \%$ and close to $30 \%$ for $\tan \lambda=5 \times 10^{5}$ and $5 \times 10^{6}$, respectively. Systematic deviations become severe when an antipodal peak becomes larger than about $20 \%$. One should choose a tradeoff parameter such that one gets velocity bounds as small as possible while avoiding systematic deviations. We take $\tan \lambda=10^{6}$ in the following analysis. There exist some small regional variations of this parameter, but they are small, and a uniform value of $\tan \lambda=10^{6}$ is optimal or near optimal everywhere on the globe for our data set.

Figures $15 a$ and $15 b$ show the kernels constructed at a point near East Pacific Rise $\left(30^{\circ} \mathrm{S}, 115^{\circ} \mathrm{W}\right)$ and a point near Azores triple junction $\left(45^{\circ} \mathrm{N}, 30^{\circ} \mathrm{W}\right)$ for Rayleigh waves at $200 \mathrm{~s}$. In both cases, the top figures are contour maps with contours at each $20 \%$ interval of maximum values at $\left(30^{\circ} \mathrm{S}, 115^{\circ} \mathrm{W}\right)$ and $\left(45^{\circ} \mathrm{N}, 30^{\circ} \mathrm{W}\right)$. Shaded regions are fast and the locations marked by cross are the places where we tried to construct a delta function. The bottom figures are three-dimensional plots of the corresponding kernels. In each case, a localized peak with a radius of about $2000 \mathrm{~km}$ is constructed. Small oscillations over the globe have amplitudes of about 3-5\% of the
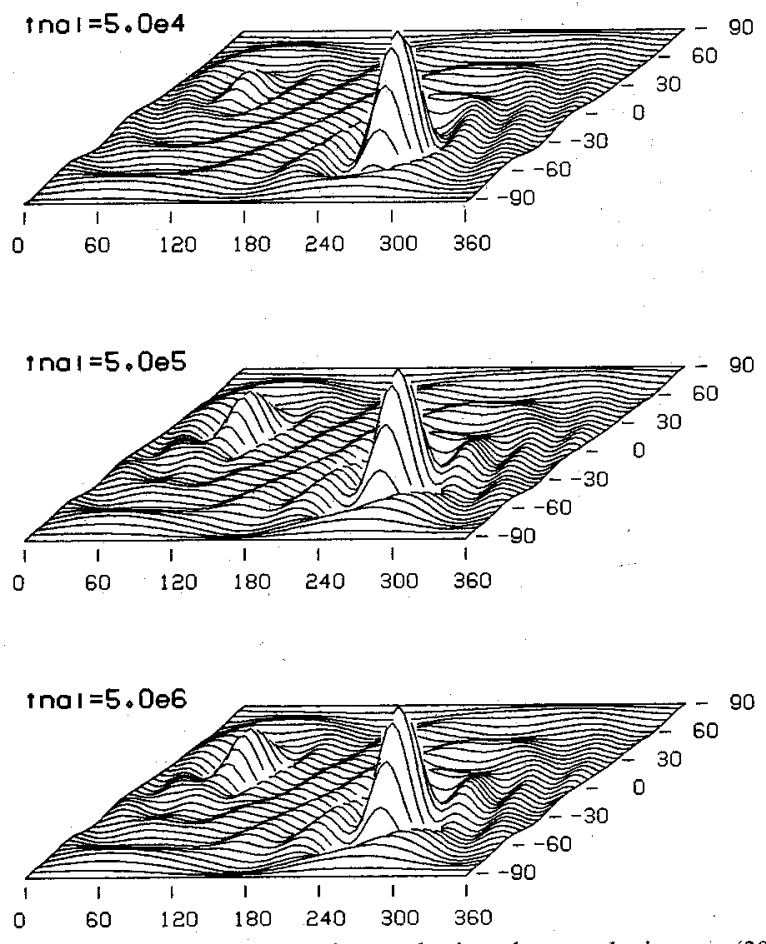

Fig. 14. Kernels constructed to obtain phase velocity at $\left(30^{\circ} \mathrm{S}\right.$, $115^{\circ} \mathrm{W}$ ) for different tradeoff parameters (tnal). 

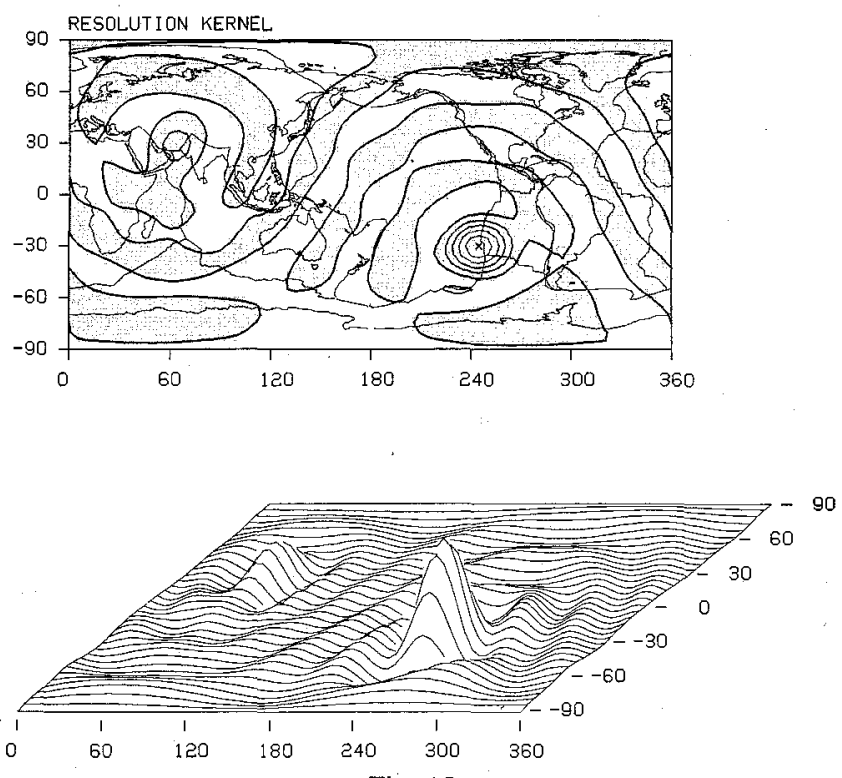

Fig. $15 a$
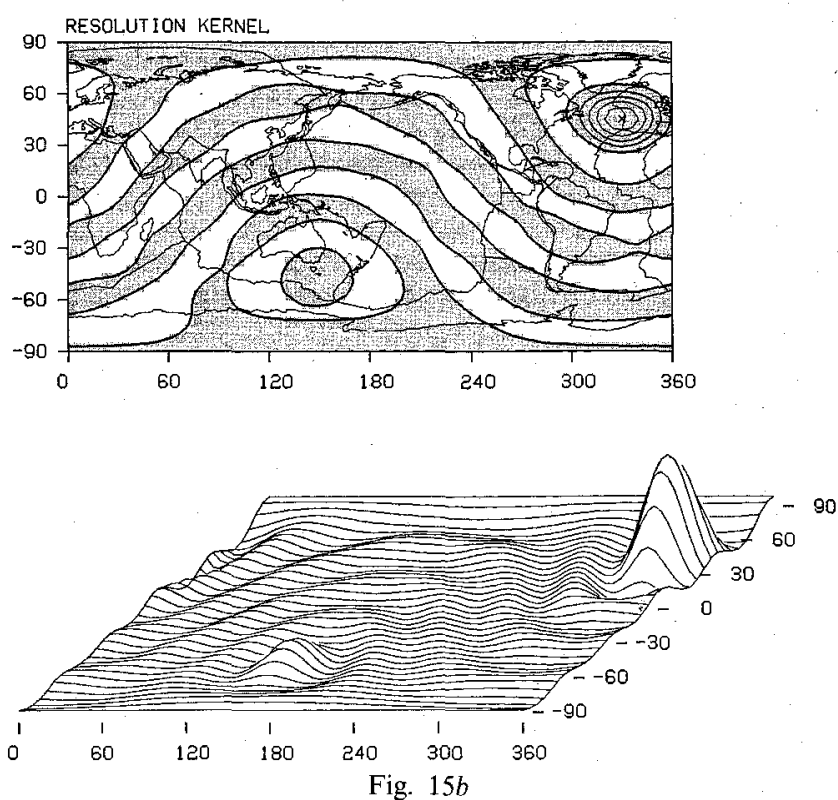

Fig. $15 b$

Fig. 15. Kernels constructed to obtain phase velocity at $(a)\left(30^{\circ} \mathrm{S}, 115^{\circ} \mathrm{W}\right)$ and $(b)\left(45^{\circ} \mathrm{N}, 30^{\circ} \mathrm{W}\right)$. Tradeoff parameter is $\tan \lambda=10^{6}$. In each case, upper figure shows contour plot at each $20 \%$ interval of the maximum value at $x$. Lower figures show three-dimerisional plot of the same kernels.

maximum peak at $x$ except at the antipodes. The peak at the antipode can become larger than $20 \%$ (Figure 15a) for some regions, which suggests that lack of constraints on odd order spherical harmonics are also the problem of the present approach. Note that this is also a problem in all previous studies [Nakanishi and Anderson, 1983, 1984; Woodhouse and Dzie- wonski, 1984]. Kernels similar to Figures $15 a$ and $15 b$ can be constructed everywhere on the globe.

Error can be estimated in the following way: statistical errors $\varepsilon^{2}$ are obtained from the coefficients $\alpha_{i}$ and the covariance matrix of the data by

$$
\varepsilon^{2}=\alpha^{t} V \alpha
$$

RAYLEIGH 200 SEC 1 PER CENT INTERVAL

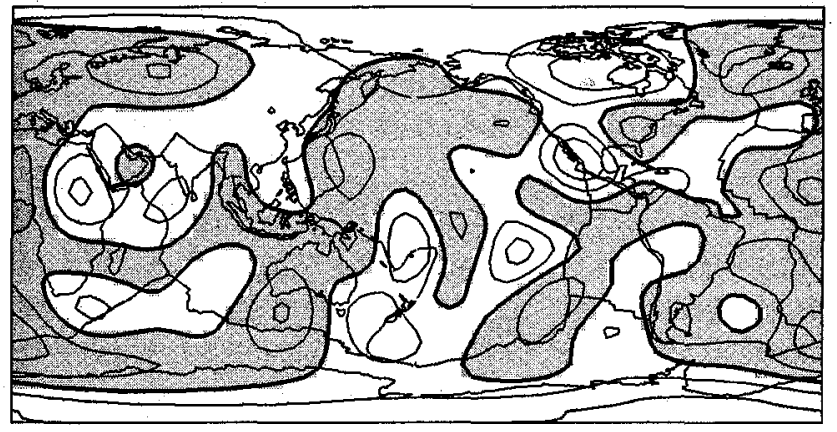

\section{ERROR 0.2 PER CENT INTERVAL}

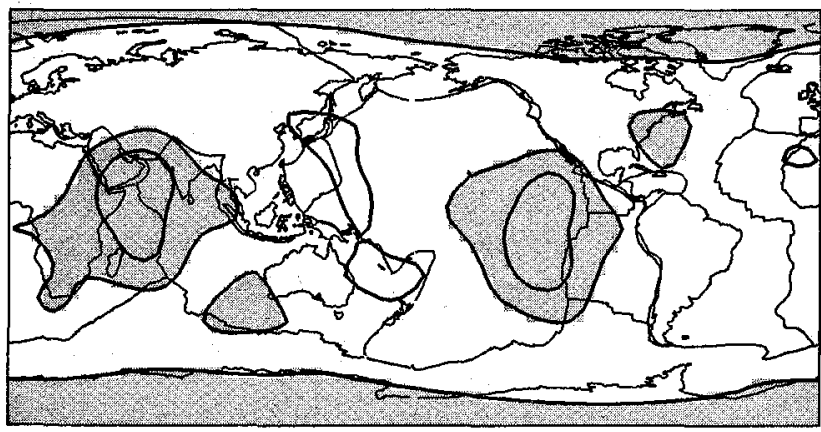

Fig. $16 a$
LOVE 200 SEC 1 PER CENT INTERVAL

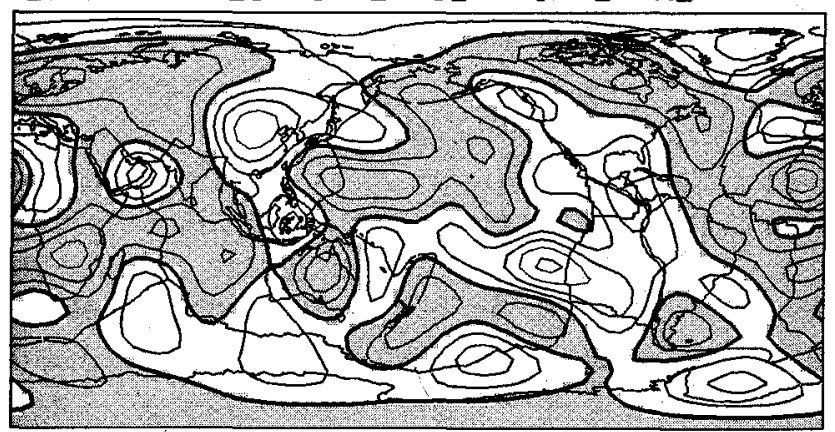

Fig. 16. Phase velocity variations with error maps for $(a)$ Rayleigh wave at $200 \mathrm{~s}$ and $(b)$ Love wave at $200 \mathrm{~s}$. Upper figures show variations of phase velocity at each $1 \%$ interval. Shaded regions denote fast velocity regions. Bottom figures show error maps at each $0.2 \%$ contour interval. Shaded regions correspond to regions with more than $1 \%$ error. These results are obtained by constructing kernels at each $5^{\circ}$ interval in longitude and latitude.

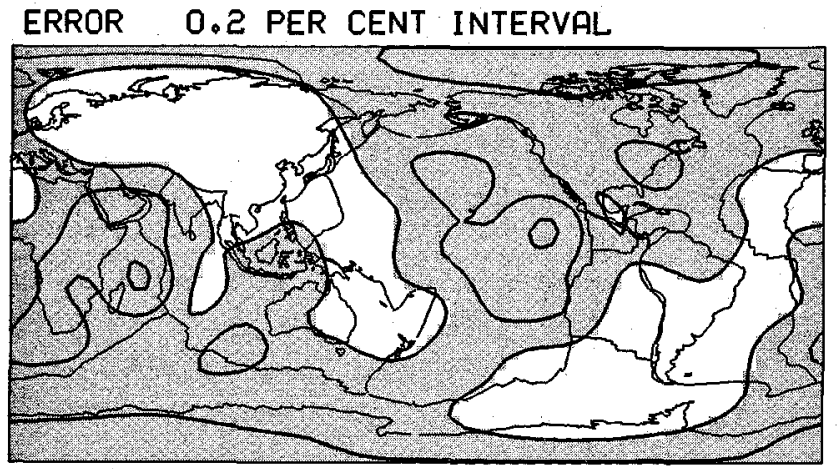

Fig. $16 b$ 
RAYLEIGH 200 SEC 1 SIGMA

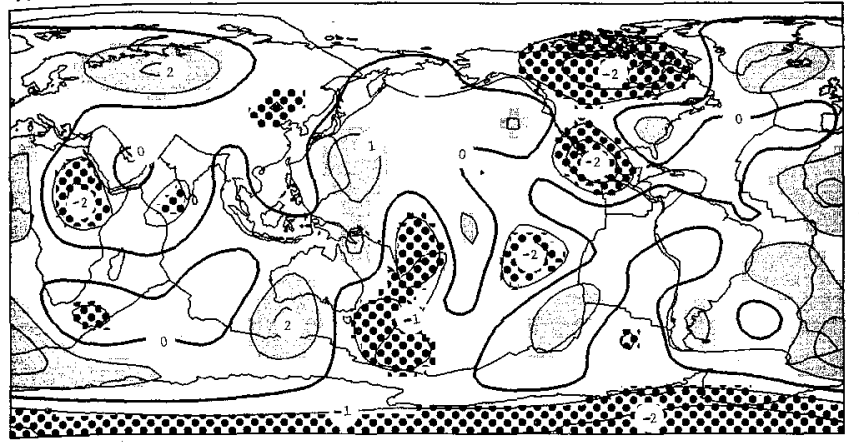

Fig. $17 a$

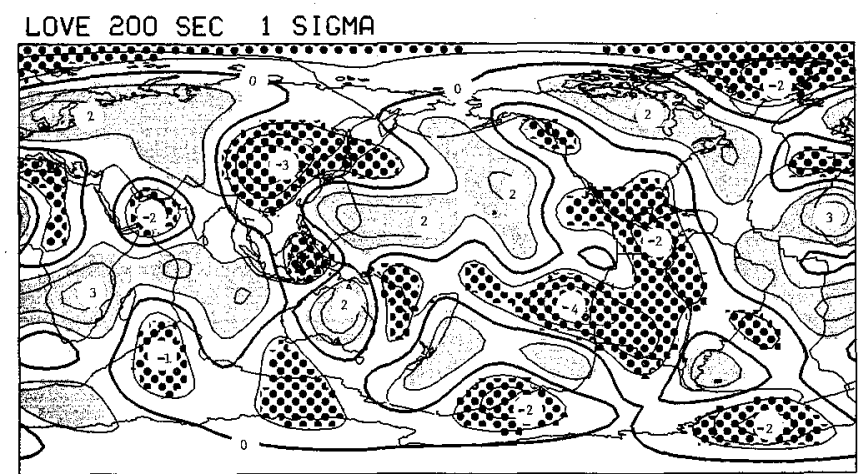

Fig. $17 b$

Fig. 17. Same as upper figures in Figures $16 a$ and $16 b$ except that absolute anomalies larger than error estimates are shown by certain patterns. Shaded regions are fast, and patterned regions are slow.

Bias in the solution is not negligible, as is clear from the shape of the kernels. Especially problematic is the antipodal peak contribution, and we estimate it as follows: Slowness estimate at a point $\left(\theta_{0, \varphi 0}\right)$ is given by

$$
\bar{S}\left(\theta_{0}, \varphi_{0}\right)=\int d \Omega K(\theta, \varphi) S(\theta, \varphi)=\int_{\text {antipode }} d \Omega K S+\int_{\text {other }} d \Omega K S
$$

Our concern is with the first term, and we estimate it by

$$
\left|\int_{\text {antipode }} d \Omega K S\right| \leq \int_{\text {antipode }} d \Omega|K||S| \leq\left|S_{\max }\right| \int_{\text {antipode }} d \Omega|K| \equiv C
$$

The integration $\int_{\text {antipode }} d \Omega|K|$ is done for the region within $20^{\circ}$ from the antipode and for $\left|S_{\max }\right|$, we use $5 \%$ of the zerothorder phase velocity. This value for $\left|S_{\max }\right|$ is pessimistic enough compared with the final results. We define the total error $\varepsilon_{\text {tot }}$ by

$$
\varepsilon_{\text {tot }} \equiv\left(\varepsilon^{2}+C^{2}\right)^{1 / 2}
$$

Figures $16 a$ and $16 b$ are the results for Rayleigh and Love waves at $200 \mathrm{~s}$, respectively. The top figures show the phase velocity variations. Shaded regions are fast velocity regions and contours are drawn at each $1 \%$ interval. These results were obtained by constructing a kernel at each $5^{\circ}$ in latitudes and longitudes. Bottom figures give error estimates $\varepsilon_{\text {tot }}$ by (11). Shaded regions in the error maps are the regions with more than $1 \%$ error and contours are drawn at each $0.2 \%$. Thus in Figure $16 a$, errors vary from slightly below $0.8 \%$ for Japan and South Pacific oceans to a little over $1.2 \%$ for East Pacific Rise and northern Indian Ocean. Errors for Love wave in Figure $16 b$ are slightly larger. Largest errors are a little over $1.4 \%$ for East Pacific Rise and northern Indian Ocean and smallest errors are somewhere between 0.8 and $1.0 \%$.

Error estimates show that phase velocity anomalies with amplitudes less than about $1 \%$ are not reliable features in the phase velocity maps. On the other hand, there exist some regions whose velocity anomalies exceed estimated errors. In Figures $17 a$ and $17 b$ these regions are filled by certain patterns. Shaded regions are fast and patterned regions are slow. Contours are again $1 \%$ intervals, and numbers give values in percent.

Comparisons between the results in this section and those in previous sections are interesting. There are similarities and discrepancies between Figure $17 a$ (Figure 16a) and Figure 9 (the third figure from top) and between Figure $17 b$ (Figure $16 b$ ) and Figure 8 (again the third from top). Note that lo- cations of fast and slow regions match quite well. However, amplitudes of anomalies do not necessarily agree. This is probably caused by the arbitrariness associated with the cutoff level of eigenvalues in Figures 8 and 9. As pointed out in a previous section, changes of the level of cutoff affect amplitudes but not the patterns.

\section{Azimuthal Dependence}

The Backus-Gilbert approach can also be used to analyze azimuthal dependence. Equation (4) can be transformed to

$$
\begin{aligned}
S_{i}=\int d \Omega\left[\bar{K}(\theta, \varphi) S(\theta, \varphi)+\bar{A}(\theta, \varphi) C_{2}(\theta, \varphi)\right. \\
\left.\quad+\bar{B}(\theta, \varphi) S_{2}(\theta, \varphi)+\bar{C}(\theta, \varphi) C_{4}(\theta, \varphi)+\bar{D}(\theta, \varphi) S_{4}(\theta, \varphi)\right],
\end{aligned}
$$

where $S(\theta, \varphi)$ is nondirectional part of phase velocity, $C_{2}(\theta, \varphi)$ and $S_{2}(\theta, \varphi)$ are $2 \Psi$ dependent parts, and $C_{4}(\theta, \varphi)$ and $S_{4}(\theta, \varphi)$ are $4 \Psi$ dependent parts. $\bar{K}, \bar{A}, \bar{B}, \bar{C}$, and $\bar{D}$ are related to paths. When assigning widths to paths, we used spherical harmonic expansion with $l \max =6$ as opposed to $l \max =10$ in the preceding section. This is because the number of parameters increases. In order to recover azimuthal dependence, kernels should be constructed for $\bar{K}, \bar{A}, \bar{B}, \bar{C}$, and $\bar{D}$ at all locations on the globe. Because of the poor coverage, however, terms with $\bar{C}$ and $\bar{D}$ are not included in the following analysis.

Figures $18 a, 18 b$, and $18 c$ show the kernels at a location in the South Pacific $\left(170^{\circ} \mathrm{E}, 2^{\circ} \mathrm{S}\right)$ for $S(\theta, \varphi), C_{2}(\theta, \varphi)$, and $S_{2}(\theta$, $\varphi$ ), respectively. This is a region with very good azimuthal path coverage, and thus localized kernels around the target location $\left(170^{\circ} \mathrm{E}, 20^{\circ} \mathrm{S}\right)$ are obtained. Trade-offs among $S(\theta, \varphi)$, $C_{2}(\theta, \varphi)$, and $S_{2}(\theta, \varphi)$ do exist but are not large. Especially striking are small trade-offs between $S(\theta, \varphi)$ and $C_{2}(\theta, \varphi)$ and between $S(\theta, \varphi)$ and $S_{2}(\theta, \varphi)$ in Figures $18 b$ and $18 c$. However, this is not the case with poorly azimuthally covered regions. Figures $19 a, 19 b$, and $19 c$ show the kernels near East Pacific Rise $\left(115^{\circ} \mathrm{W}, 20^{\circ} \mathrm{S}\right)$. Figures $19 b$ and $19 c$ show clearly that our data cannot resolve azimuthal dependence in this region.

We found from further analysis of kernels that the current data can resolve the azimuthal dependence in an azimuthally very well covered region, e.g., southern Europe, Japan, South Pacific, western North America, and northern and southern Atlantic for Rayleigh waves (Figure 1) but not elsewhere. Much more data are required for the analysis of the entire globe. However, one should note that this does not necessarily prove that low angular order spherical harmonic components like $l=1-4$ are not obtainable from the data set, since the 
THETA $=110 \quad \mathrm{PHI}=170$

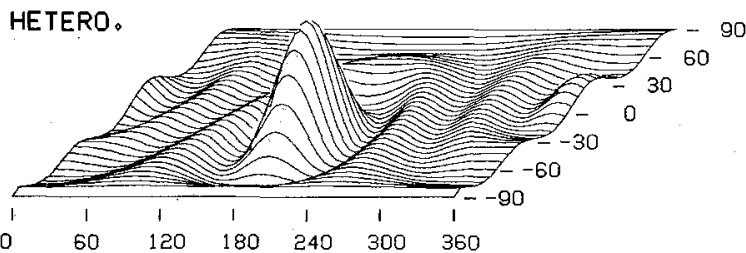

$\cos (2 \mathrm{PSI})$

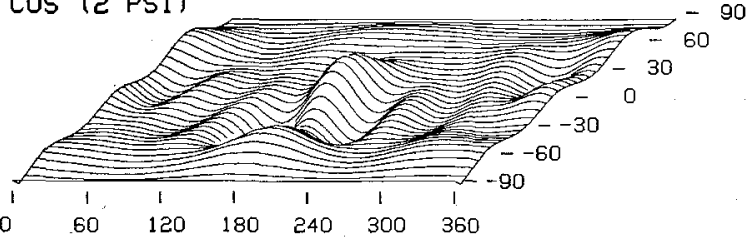

\section{SIN (2 PSI)}

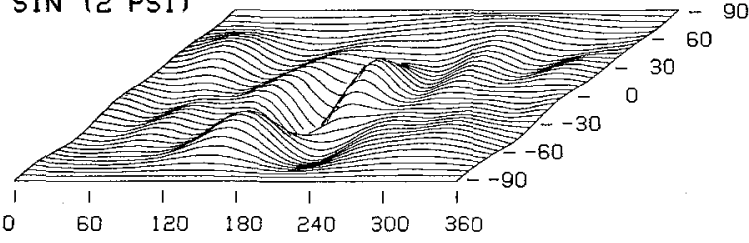

THETA $=110 \quad \mathrm{PHI}=170$

HETERO。

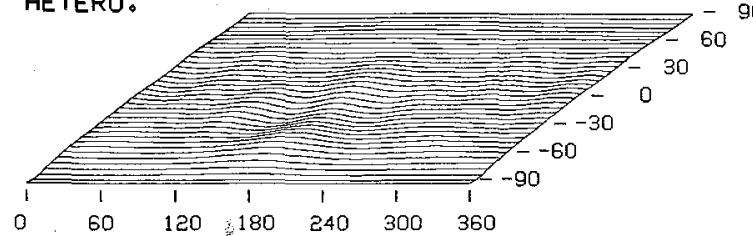

$\cos (2 \mathrm{PSI})$

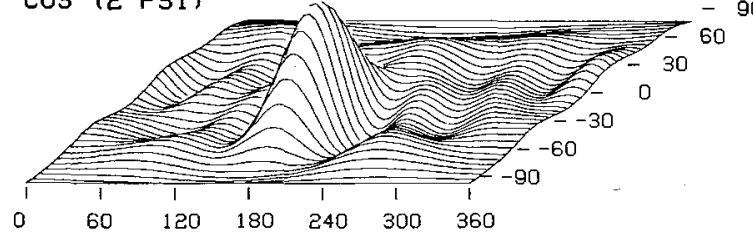

THETA $=110 \quad \mathrm{PHI}=170$
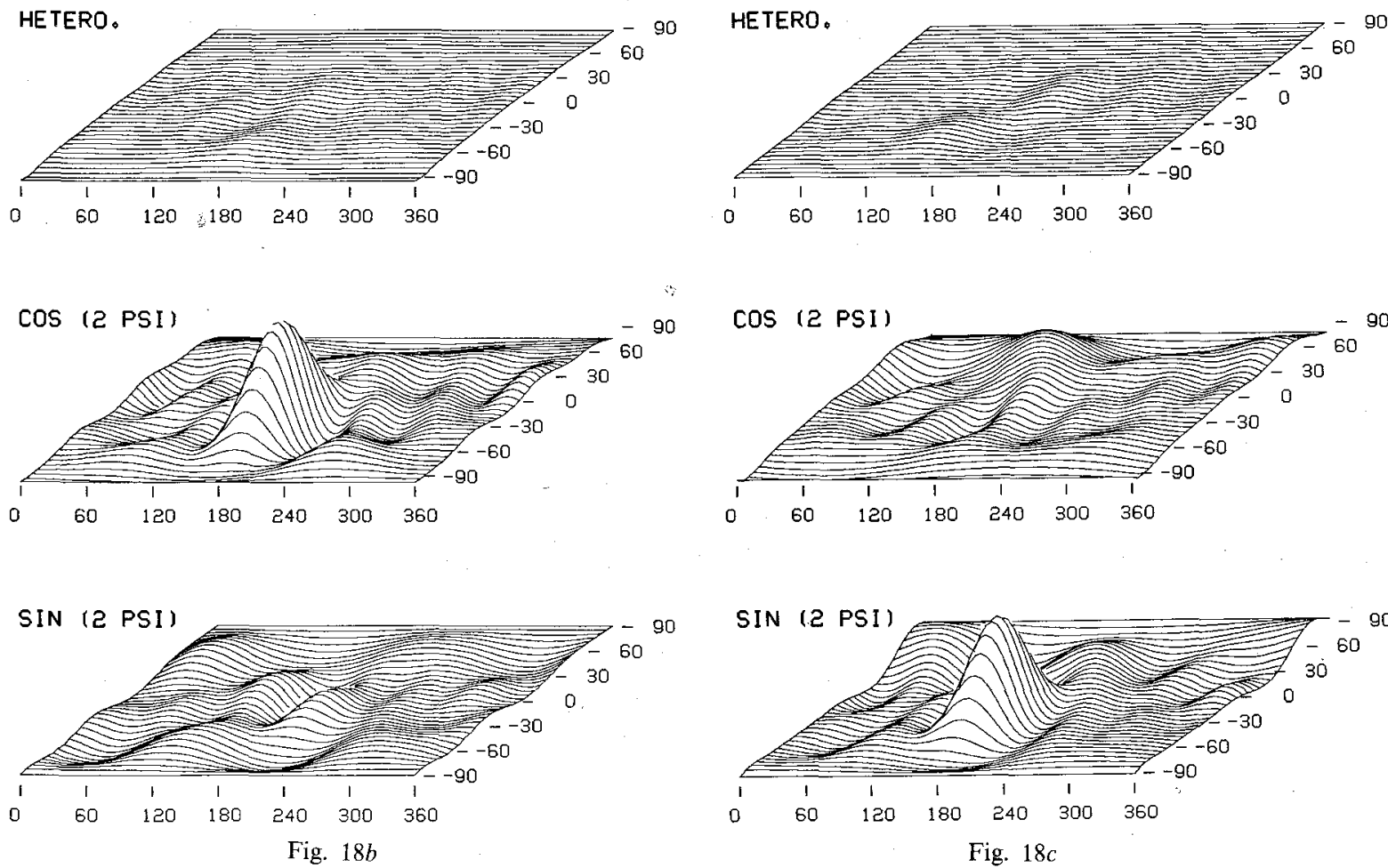

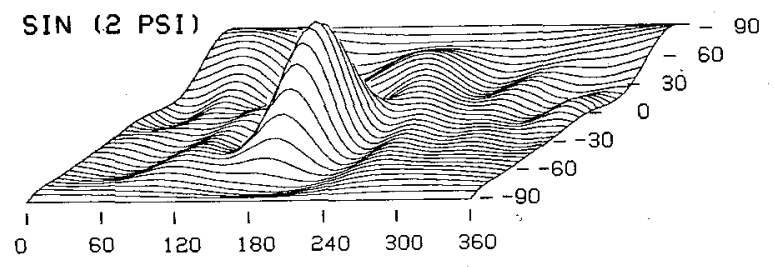

Fig. $18 c$

Fig. $18 a$

Fig. 18. Resolving kernels constructed to obtain $(a)$ nondirectional part of phase velocity, $(b) \cos 2 \Psi$ dependent part, and $(c)$ sin $2 \Psi$ dependent part. This is at $\left(20^{\circ} \mathrm{S}, 170^{\circ} \mathrm{E}\right)$. Note the small trade-offs between nondirectional part and $2 \Psi$ dependent parts in Figures $18 b$ and $18 \mathrm{c}$. 
THETA $=120 \quad \mathrm{PHI}=245$

\section{HETERO。}

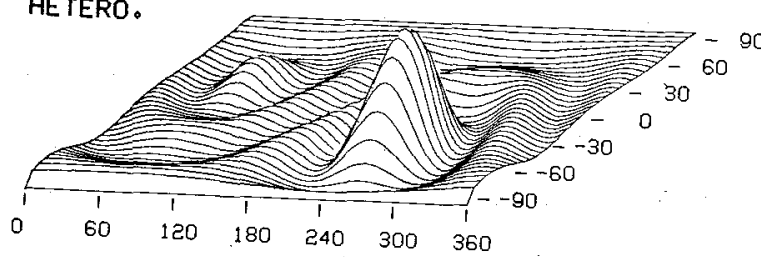

$\cos (2$ PSI)

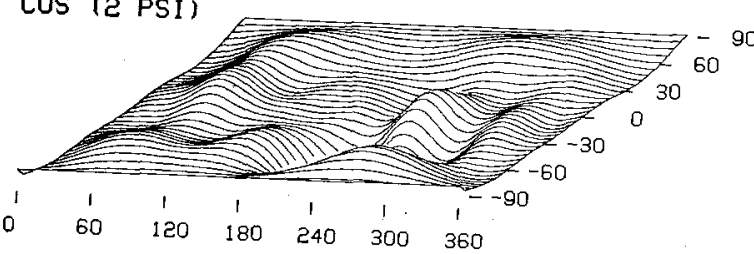

SIN (2 PSI)

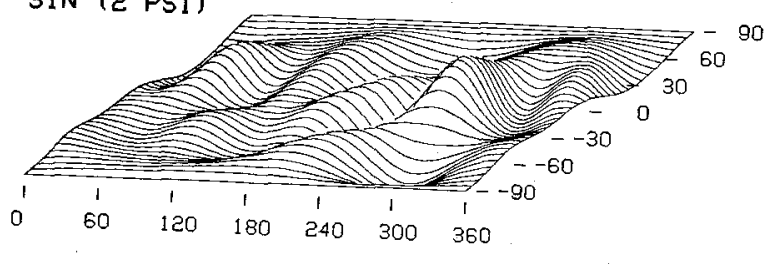

Fig. $19 a$
THETA $=120 \quad \mathrm{PHI}=245$

HETERO。
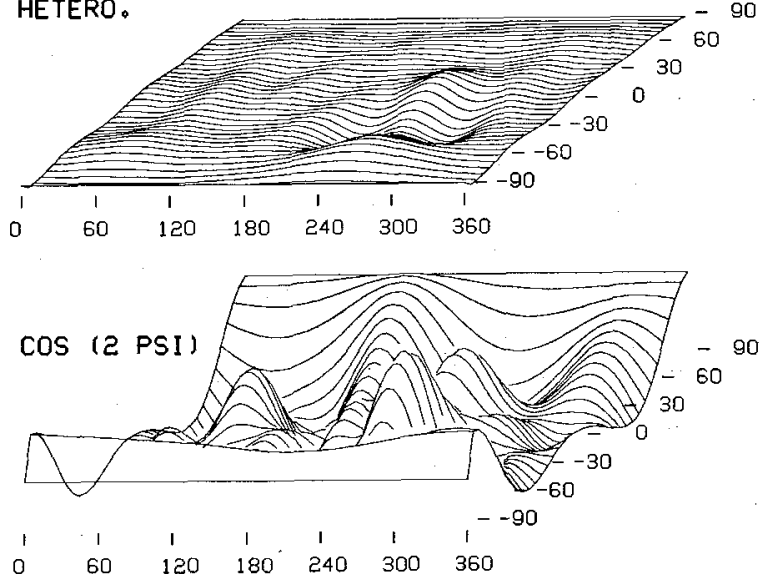

$\begin{array}{ccccccc}1 & 1 & 1 & 1 & 1 & 1 & 1^{-}-90 \\ 0 & 60 & 120 & 180 & 240 & 300 & 360\end{array}$

SIN (2 PSI)

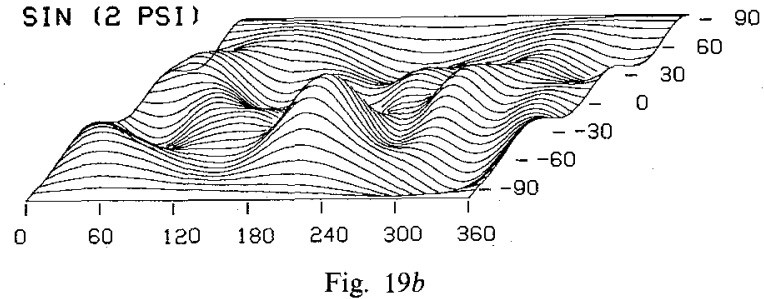

THETA $=120$

$\mathrm{PHI}=245$
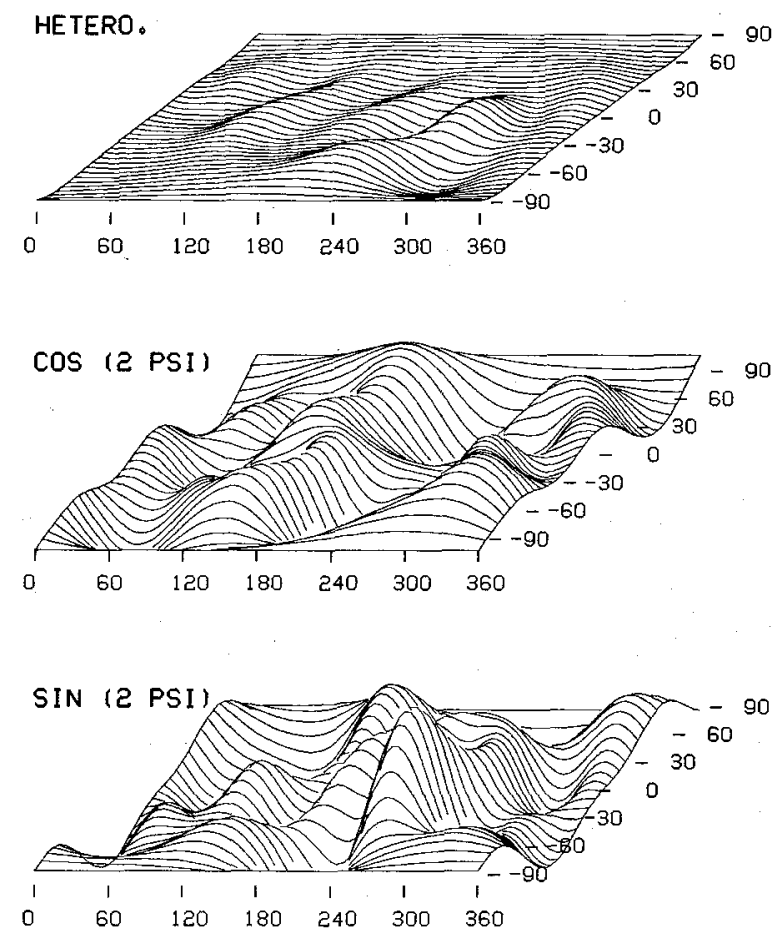
Fig. $19 c$

Fig. 19. Same as Figure 18 except that this is a different location $\left(30^{\circ} \mathrm{S}, 115^{\circ} \mathrm{W}\right)$. Figures $19 b$ and $19 c$ clearly show that the data set can not resolve directional dependence at this point. 
present analysis uses the deltaness criterion for a particular region and in essence requires much higher angular order components. We do not discuss this point further in this paper. More reliable conclusions will be reached when we collect more data.

\section{Conclusions}

Using phase velocities measured for 15 earthquakes in 1980 , lateral variation of phase velocity and its azimuthal dependence were analyzed. Two methods, spherical harmonic approach with $l \max =10$ and the Backus-Gilbert method are used. In both methods, slow regions are evident at the East Pacific Rise, northeast Africa, Tibet, Tasman Sea, southwestern North America, and triple junctions in the northern Atlantic and Indian oceans. Fast regions occur in Australia, western Pacific, and southern Atlantic.

Resolution kernels with a radius of about $2000 \mathrm{~km}$ can be constructed for the present data set. The estimated errors are about $1 \%$ of the phase velocity in the zeroth-order spherically symmetric earth model. A factor of about 2 variations of errors are obtained with maximum values occurring near the East Pacific Rise and northern Indiarr Ocean.

Fast phase velocity directions which seem to correlate well with plate motion vectors are obtained for low angular order spherical harmonics, $l=1-4$. The resolution analysis by the Backus-Gilbert method shows, however, that $2 \Psi$ dependence can be resolved from the present data set only in azimuthally well-covered regions. A few features in the solution, e.g., NW-SE fast direction in the Pacific and NNE-SSW fast direction in the west of Australia, seem to be stable. Although this does not necessarily prove that the present data cannot resolve low angular order components of the spherical harmonic expansions, more complete data are required for confirmation.

Acknowledgments. We wish to thank Ichiro Nakanishi for listening to our ideas and kindly supplying his data. The IDA data used were made available to us by courtesy of the IDA project team at the Institute of Geophysics and Planetary Physics, University of California. This research was supported by National Science Foundation grants EAR-8115236 and EAR-8317623 and NASA grant NSG-7610. Contribution 3993, Division of Geological and Planetary Sciences, California Institute of Technology, Pasadena, California.

\section{REFERENCES}

Anderson, D. L., and A. M. Dziewonski, Upper mantle anisotropy: Evidence from free oscillations, Geophys. J. R. Astron. Soc., 69, 383-404, 1982.

Anderson, D. L., and J. Regan, Upper mantle anisotropy and the oceanic lithosphere, Geophys. Res. Lett., 10, 841-844, 1983.
Backus, G. E., Possible forms of seismic anisotropy of the uppermost mantle under oceans, J. Geophys. Res., 70, 3429-3439, 1965.

Backus, G. E., and F. Gilbert, Numerical application of a formalism for geophysical inverse problems, Geophys. J. R. Astron. Soc., 13, 247-276, 1967.

Backus, G. E., and F. Gilbert, The resolving power of gross earth data, Geophys. J. R. Astron. Soc., 16, 169-205, 1968.

Backus, G. E., and F. Gilbert, Uniqueness in the inversion of inaccurate gross earth data, Philos. Trans. R. Soc. London Ser. A, 266, 185-294, 1970.

Dziewonski, A. M., and D. L. Anderson, Travel times and station corrections for $P$ waves at teleseismic distances, J. Geophys. Res., $88,3295-3314,1983$.

Masters, G., T. H. Jordan, P. G. Silver, and F. Gilbert, Aspherical earth structure from fundamental spheroidal-mode data, Nature, $298,609-613,1982$.

Nakanishi, I., and D. L. Anderson, World-wide distribution of group velocity of mantle Rayleigh waves as determined by spherical harmonic inversion, Bull. Seismol. Soc. Am., 72, 1185-1194, 1982.

Nakanishi, I., and D. L. Anderson, Measurements of mantle wave velocities and inversion for lateral heterogeneity and anisotropy, 1 , Analysis of great circle phase velocities, J. Geophys. Res., 88, 10267 10283,1983 .

Nakanishi, I., and D. L. Anderson, Measurements of mantle wave velocities and inversion for lateral heterogeneity and anisotropy, 2, Analysis by the single-station method, Geophys. J. R. Astron. Soc., 78, 573-617, 1984.

Nakanishi, I., and H. Kanamori, Source mechanisms of twenty-six large shallow earthquakes $\left(M_{s}>6.5\right)$ during 1980 from $P$-wave first motion and long-period Rayleigh wave data, Bull. Seismol. Soc. Am., 74, 805-818, 1984

Silver, P. G., and T. H. Jordan, Fundamental spheroidal mode observations of aspherical heterogeneity, Geophys. J. R. Astron. Soc., 64, 605-634, 1981.

Smith, M. L., and F. A. Dahlen, The azimuthal dependence of Love and Rayleigh wave propagation in a slightly anisotropic medium, J. Geophys. Res., 78, 3321-3333, 1973.

Sobel, P. A., and D. H. von Seggern, Applications of surface-wave ray tracing, Bull. Seismol. Soc. Am., 68, 1359-1380, 1978.

Whaler, K. A., and D. Gubbins, Spherical harmonic analysis of the geomagnetic field: An example of a linear inverse problem, Geophys. J. R. Astron. Soc., 65, 645-693, 1981.

Woodhouse, J. H., The joint inversion of seismic waveforms for lateral variations in Earth structure and earthquake source parameters, Proc. Int. Sch. Phys. Enrico Fermi, 85, 366-397, 1984.

Woodhouse, J. H., and A. M. Dziewonski, Mapping the upper mantle: Three-dimensional modeling of earth structure by inversion of seismic waveforms, J. Geophys. Res., 89, 5953-5986, 1984.

D. L. Anderson and T. Tanimoto, Seismological Laboratory, California Institute of Technology, MS/252-21, Pasadena, CA 91125.

(Received October 24, 1983; revised June 7, 1984; accepted October 19, 1984.) 